\title{
Phase boundary dynamics: transition between ordered and disordered lipid monolayers
}

\author{
Hans Wilhelm Alt and Wolfgang Alt \\ Applied Mathematics and Theoretical Biology, University of Bonn, D-53115 Bonn, Germany \\ E-mail: H.W.Alt@t-online.de
}

[Received 11 November 2007 and in revised form 6 June 2008]

\begin{abstract}
Based on a general thermodynamical theory of mass and momentum, we propose and investigate a new phase field model for small transition layers between two spatially separated phases with intersecting free energy functions. We use a phase fraction that only depends on the ratio of the two density components. From the phase field model we derive conditions for the sharp interface velocity and density jumps.

The general model is motivated by and applied to the dynamics of lipid monolayers, which appear as surfactant on the strongly expanded and compressed thin water film of lung alveoli. While the liquid condensed ordered phase (LC) of a flat lipid monolayer is characterized by high viscosity and limited compressibility, the liquid expanded disordered phase (LE) is dominated by diffusion and high compressibility.

In order to perform the asymptotic transition layer analysis at moving phase boundaries, a new nonlinear free energy interpolation model is proposed whose excess energy, in comparison to standard linear interpolations, contains an energy hump that has to be surpassed in a permissive transition from one phase to the other. This leads to a unique density jump condition in the case that the ordered phase is extending, whereas in the retracting case the jump densities are not restricted. The transition profiles and the resulting interface speed are numerically determined for a typical example by solving a nonlinear degenerate ODE system.

In a simplified 1-dimensional situation with low Reynolds number, the approximate macroscopic system of differential equations with moving sharp interface is numerically solved and interpreted in application to surfactant monolayers in lung alveoli.
\end{abstract}

\section{Introduction: Lipid layers as surfactant in lung alveoli}

Surfactant layers on the top of the thin water film covering the interior of lung alveoli consist of a well tuned mixture of phospholipids (with 40-80\% DPPC: dipalmitoylphosphatidylcholine) and associated proteins, whose hydrophilic and hydrophobic interactions guarantee film stability by effectively reducing water-air surface tension even under extreme changes of alveolar surface area and lateral pressure due to strong breathing (see [8]).

In vivo and in vitro studies have revealed that during repetitive breathing cycles, depending on the lipid mixture, there appear more or less variable surface patches of well ordered lipid monolayers, while the remaining surface is covered either by folded multilayer 'islands' or by regions of highly disordered and compressible lipid layers. Within these three types of patches, lipid concentration levels are relatively constant, whereas at the patch boundaries there can occur quite steep concentration jumps (see Fig. 1).

Though the type of lipid mixture as well as interactions with embedded proteins play an enhancing role for surface stability, we only treat the simplest case of a pure DPPC lipid monolayer. 

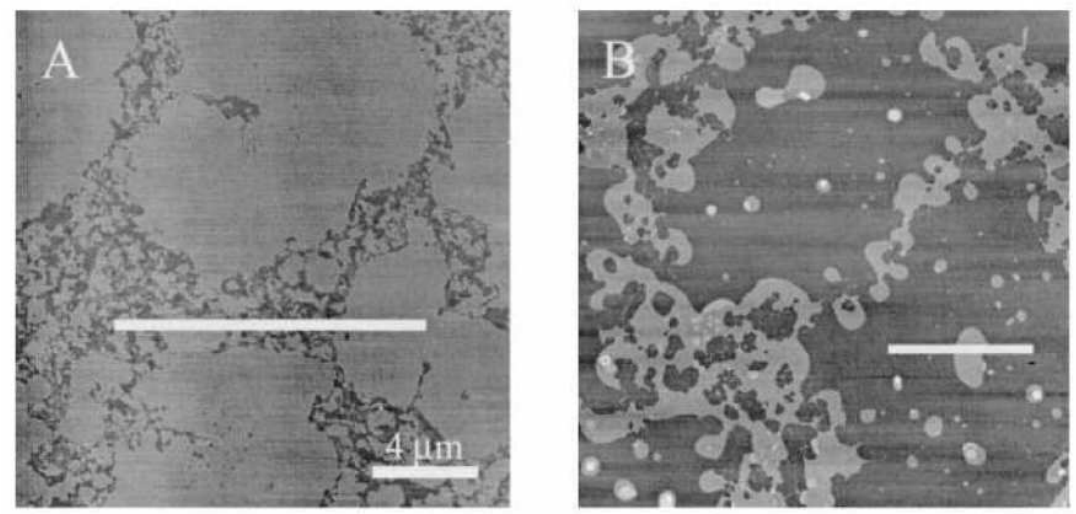

$\ddot{\circ}_{\dot{0}}^{\mathrm{m}}$

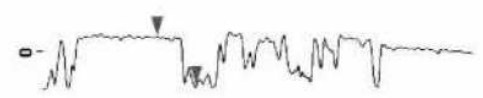

i⿱⺈

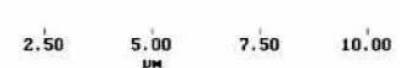

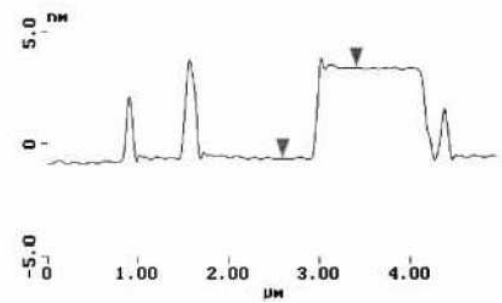

FIG. 1. Atomic force microscopic images of lung surfactant monolayers on an air-water surface at pressures (A) $45 \mathrm{nN} / \mathrm{m}$ and (B) $55 \mathrm{nN} / \mathrm{m}$. The displayed layer height (scale $5 \mathrm{~nm}$ ) appearing as grey light level, reveals jump transitions of different sign between mostly constant plateau values within the larger (5-10 $\mu \mathrm{m}$-sized), more circular 'solid phase' domains and smaller, more fuzzy 'liquid phase' domains. From Ding et al. [4], Fig. 2.

(a)

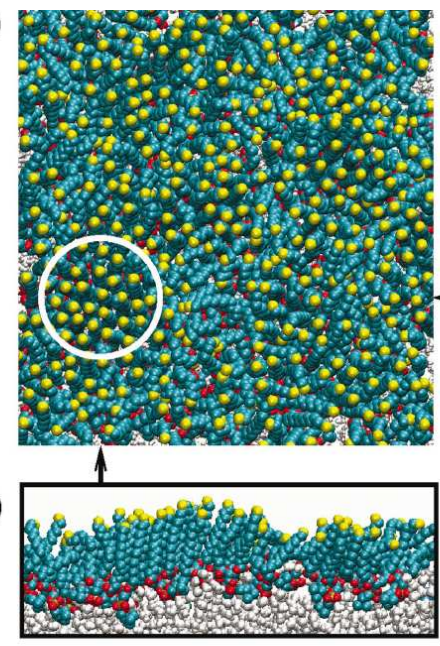

(c)

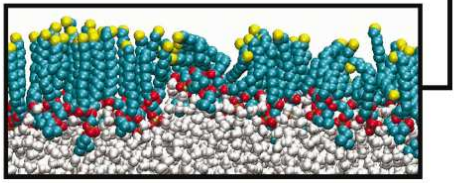

FIG. 2. Phospholipid monolayer simulated by molecular dynamics: Larger patch $(10.7 \mathrm{~nm} \times 311.6 \mathrm{~nm})$ in the $\mathrm{LE}+\mathrm{LC}$ coexistence region $\left(0.57 \mathrm{~nm}^{2} /\right.$ molecule $)$ after $7.8 \mathrm{~ns}$ of simulation time. (a): View from the air phase. The white circle marks a LC domain, indicated by a hexagonal arrangement of the terminal carbon atoms of lipid tails (bright). (b) and (c): Side views of slices along the straight lines indicated by the arrows. From Knecht et al. [5], Fig. 5. 
Moreover, we do not consider the process of multilayer formation by monolayer folding, but restrict our modeling to a flat 2-dimensional situation with relatively rapid transitions at the phase boundaries between ordered and disordered lipid monolayers.

Also 3-dimensional molecular dynamics simulations have been performed for densely packed arrays of phospholipids at an air-water interface, where the hydrophilic heads are embedded into the water (Knecht et al. [5]). Under certain hypotheses about the head/tail specific molecular interactions, the authors are able to reproduce some phenomena of molecular orientation, ordering and patch formation that resemble phase transition and, in a few cases, also phase segregation between more ordered and less ordered states (see Fig. 2). However, the presented spatial scaling might only indirectly be applicable to the $\mu \mathrm{m}$-scale in the situations of Fig. 11, which rather require suitable macroscopic continuum descriptions.

Our goal is to understand and explain the basic processes at the molecular level that lead to macroscopic phase boundaries. For this we derive and exploit a generic phase field model for small continuous transition layers. In the limit we obtain equations with jump discontinuities, which are of different type in cases of an expanding or retracting ordered monolayer phase.

\section{Model functions for ordered and disordered lipid monolayers}

According to experimental investigations of pure DPPC monolayers on flat air-water interfaces under controlled lateral compression, there appear two essentially different liquid phases, namely an ordered gel-like liquid condensed phase (LC) at about 2 molecules per $\mathrm{nm}^{2}$, where the hydrophobic lipid tails interact and induce their mutual alignment, with the hydrophilic lipid heads being 'anchored' in water at a relatively constant depth, and a disordered liquid expanded phase (LE) at about 1 molecule per $\mathrm{nm}^{2}$, where the lipid tails do not align but rather cross each other, partially floating on the water surface, thereby diffusing together with their "less anchored' lipid heads in a gas-like manner $(\mathrm{G})$, particularly at even lower concentrations (see Fig. 33. At intermediate concentrations the disordered and ordered phases show coexistence (LE+LC), whereas for a 'maximal' concentration slightly above 2 molecules per $\mathrm{nm}^{2}$ a perfectly ordered and aligned condensed phase (C) arises, which tends to collapse into folded 3-dimensional structures.

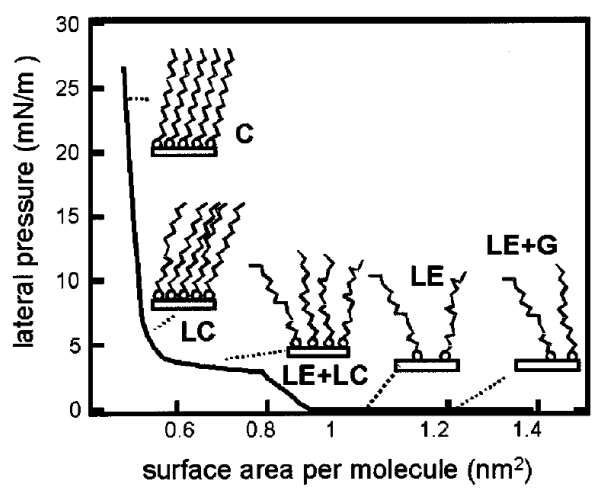

FIG. 3. Phase behaviour in experimental DPPC lipid monolayers on air-water interfaces. Sketch of observed relations between lateral pressure and inverse concentration (surface area per lipid molecule). From Knecht et al. [5], Fig. 1. 
The disordered phase 1 ( $\mathrm{LE}$ or $\mathrm{LE}+\mathrm{G})$ is characterized by a nearly constant lateral pressure, so that a gas-like pressure state function, e.g. of the simple form (up to a constant)

$$
p_{1}(\varrho)=a_{1} \cdot\left(\varrho^{2}-\varrho_{1}^{* 2}\right)+b_{1} \cdot\left(\varrho-\varrho_{1}^{*}\right)-c_{1}
$$

with relatively small coefficients, is suggested. Then the corresponding free energy function (see Section 3] can be written as

$$
f_{1}(\varrho)=a_{1} \cdot\left(\varrho-\varrho_{1}^{*}\right)^{2}+b_{1} \cdot \varrho \cdot\left(\ln \frac{\varrho}{\varrho_{1}^{*}}-1\right)+b_{1} \cdot \varrho_{1}^{*}+c_{1}
$$

where $c_{1}$ denotes the minimal free energy which is attained at a concentration $\varrho=\varrho_{1}^{*}$. From the experimental diagram in Fig. 3 , values of $\varrho_{1}^{*} \leqslant 1 / \mathrm{nm}^{2}$ would be realistic.

For increasing condensation the ordered phase 2 (LC or LE+LC) is characterized by a rapidly increasing pressure tending to infinity for $\varrho=\varrho_{\max }$, with $\varrho_{\max } \sim 2.5 / \mathrm{nm}^{2}$. This functional dependence can be modeled as

$$
p_{2}(\varrho)=a_{2} \cdot \varrho \cdot \frac{\varrho_{\max }}{\varrho_{\max }-\varrho}+b_{2} \cdot\left(\varrho-\varrho_{2}^{*}\right)-c_{2}
$$

corresponding to a free energy function

$$
f_{2}(\varrho)=a_{2} \cdot \varrho \cdot \ln \frac{\varrho}{\varrho_{\max }-\varrho}+b_{2} \cdot \varrho \cdot\left(\ln \frac{\varrho}{\varrho_{2}^{*}}-1\right)+b_{2} \cdot \varrho_{2}^{*}+c_{2}
$$

where now $c_{2}$ would denote the minimal free energy at $\varrho=\varrho_{2}^{*}$ in case of lipid alignment without any specific force interaction between the tails $\left(a_{2}=0\right)$, just as gas-like repulsion between the heads. Therefore, in comparison to the free energy function of the disordered phase 1, with repulsion of heads and tails, we propose basic parameter choices $\varrho_{2}^{*}>\varrho_{1}^{*}, b_{2}>b_{1}$ and $c_{2}<c_{1}$, such that already for $a_{1}=a_{2}=0$ the ordered phase 2 has a steeper free energy profile with a lower absolute minimum attained at higher concentrations (see Fig. 4). We remark that $c_{1}$ and $c_{2}$ are only defined up to a common additive constant $C$, which has to be chosen so that the pressure in the disordered phase vanishes for $\varrho \rightarrow 0$ (in Fig. 4 choose $C=-3$ ).
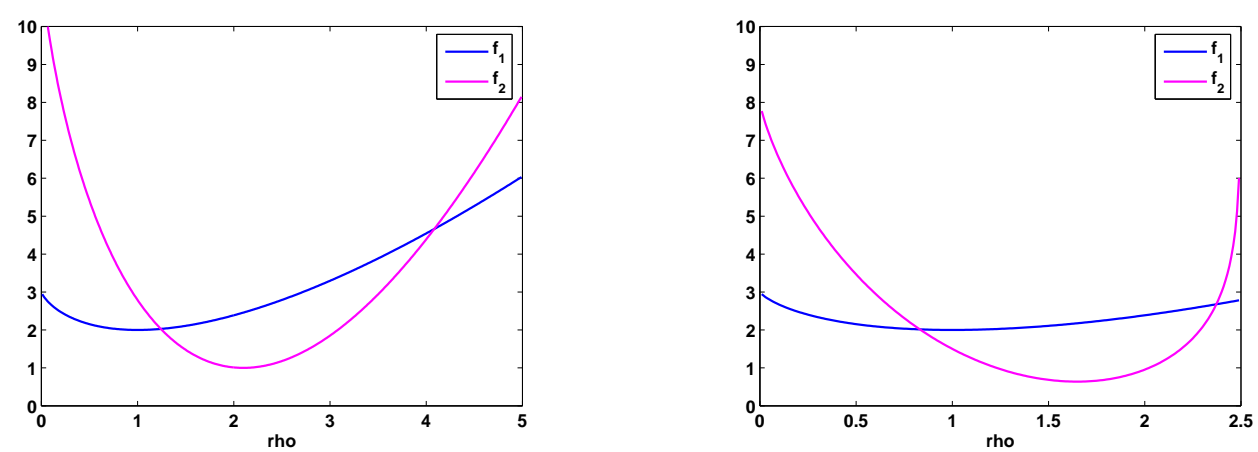

FIG. 4. Modeled free energy functions $f_{1}$ and $f_{2}$ plotted over mixture density $\varrho$ for the disordered and ordered phases 1 and 2: (a) Left panel: Gibbs energies with parameters $a_{1}=a_{2}=0, \varrho_{1}^{*}=1$ and $\varrho_{2}^{*}=2.1, b_{1}=1$ and $b_{2}=5, c_{1}=2$ and $c_{2}=1$. (b) Right panel: Generalization with $a_{1}=0, a_{2}=0.5, \varrho_{1}^{*}=1$ and $\varrho_{2}^{*}=3$, as well as $b_{1}=1$ and $b_{2}=3, c_{1}=2$, $c_{2}=-1$, and $\varrho_{\max }=2.5$. 


\section{Interface problem}

Let us consider the local situation without boundary conditions, in which a time-space domain $\Omega \subset \mathbb{R} \times \mathbb{R}^{n}$ is decomposed into two domains $\Omega^{1}, \Omega^{2}$ separated by an interface $\Gamma \subset \mathbb{R} \times \mathbb{R}^{n}$. For the physical situation the space dimension is $n=2$. We assume that $\Omega^{1}$ is occupied by a diffusive substance with density $\varrho_{1}$ and low viscosity, and that $\Omega^{2}$ is occupied by an almost incompressible substance with density $\varrho_{2}$ and high viscosity. Moreover, we assume that two strictly convex free energies

$$
\varrho_{1} \mapsto f_{1}\left(\varrho_{1}\right) \quad \text { (disordered phase), } \varrho_{2} \mapsto f_{2}\left(\varrho_{2}\right) \quad \text { (ordered phase), }
$$

are given (for examples see Fig. 4 and Fig. 57).

The dynamics is given by conservation of mass and momentum for the mass densities $\varrho_{1}$ and $\varrho_{2}$ defined in $\Omega^{1}$ and $\Omega^{2}$, and a velocity $v$ defined in $\Omega$ and assumed to be continuous at the interface $\Gamma$, since it describes the mean flow field of the fluid mixture.

Denoting by $\mathbf{m}_{\Omega}$ and $\mathbf{m}_{\Omega^{k}}$ the volume measure $L^{1} \times L^{n}$ on $\Omega$ and $\Omega^{k}$, and by $\mathbf{m}_{\Gamma}$ the surface measure $L^{1} \times H^{n-1}$ on $\Gamma$, the distributional formulation (see [1]) is given by

3.1. Conservation laws. The balance laws for the mass of the two phases are

$$
\begin{aligned}
\partial_{t}\left(\varrho_{1} \mathbf{m}_{\Omega^{1}}\right)+\operatorname{div}\left(\left(\varrho_{1} v+J\right) \mathbf{m}_{\Omega^{1}}\right) & =r_{1} \mathbf{m}_{\Gamma}, \\
\partial_{t}\left(\varrho_{2} \mathbf{m}_{\Omega^{2}}\right)+\operatorname{div}\left(\left(\varrho_{2} v\right) \mathbf{m}_{\Omega^{2}}\right) & =r_{2} \mathbf{m}_{\Gamma},
\end{aligned}
$$

with conserved total mass, that is, $r_{1}+r_{2}=0$ for the sum of the two mass transition rates $r_{i}$ at the interface. Here $J$ is the diffusive flux of the disordered phase, for a linear diffusion model given by $J=-\tilde{d}\left(\varrho_{1}\right) \nabla \varrho_{1}$ or, equivalently, by

$$
J=-d\left(\varrho_{1}\right) \nabla \mu_{1}, \quad \text { where } \quad \mu_{1}:=f_{1}^{\prime}\left(\varrho_{1}\right)
$$

is the chemical potential of the disordered phase. The balance law for the total momentum is

$$
\partial_{t}\left(\varrho v \mathbf{m}_{\Omega}\right)+\operatorname{div}\left(\varrho v \otimes v \mathbf{m}_{\Omega}+v \otimes J \mathbf{m}_{\Omega^{1}}+\sum_{k=1,2} \Pi^{k} \mathbf{m}_{\Omega^{k}}\right)=\mathbf{f} \mathbf{m}_{\Omega},
$$

where $\varrho:=\varrho_{1}$ in $\Omega^{1}$ and $\varrho:=\varrho_{2}$ in $\Omega^{2}$, and where $v$ is continuous across the interface. (Here the splitting of the momentum flux is in accordance with general thermodynamical considerations.) Moreover, we set

$$
\Pi^{k}=p_{k} \mathrm{Id}-S_{k}
$$

with pressures $p_{k}=\varrho_{k} f_{k}^{\prime}\left(\varrho_{k}\right)-f_{k}\left(\varrho_{k}\right)$ and stress tensors $S_{k}$, for which we use standard constitutive relations for fluids, namely

$$
S_{k}=\alpha_{k}\left(\varrho_{k}\right)(D v)^{\mathrm{S}}+\beta_{k}\left(\varrho_{k}\right) \operatorname{div} v \mathrm{Id} .
$$

Finally, $\mathbf{f}$ is a possible external force.

This is the complete formulation of the problem, with one exception: an additional equation on the interface is needed. It is the purpose of this paper (see 3.2, to arrive at such constitutive equations. Now, the equations in (3.2) are equivalent to

$$
\begin{array}{ll}
\partial_{t} \varrho_{1}+\operatorname{div}\left(\varrho_{1} v+J\right)=0 & \text { in } \Omega^{1} \text { (parabolic), } \\
\partial_{t} \varrho_{2}+\operatorname{div}\left(\varrho_{2} v\right)=0 & \text { in } \Omega^{2} \text { (hyperbolic), }
\end{array}
$$


and the interface conditions

$$
r_{1}+\left(\varrho_{1} \cdot\left(v-v_{\Gamma}\right)+J\right) \bullet v_{\Omega^{1}}=0, \quad r_{2}+\varrho_{2} \cdot\left(v-v_{\Gamma}\right) \bullet v_{\Omega^{2}}=0,
$$

where $v_{\Gamma}$ is the velocity vector of $\Gamma$. Since $r_{1}+r_{2}=0$, we obtain one interface condition

$$
-J \cdot v+\lambda \cdot\left(\varrho_{1}-\varrho_{2}\right)=0,
$$

where $v:=v_{\Omega^{1}}=-v_{\Omega^{2}}$ denotes the interface normal vector and

$$
\lambda:=\left(v_{\Gamma}-v\right) \bullet v
$$

is the speed of the interface in direction of the ordered phase relative to the normal component of the fluid velocity. Thus the two counteracting mass transition rates are

$$
r_{1}=\varrho_{1} \lambda-J \bullet v \quad \text { and } \quad r_{2}=-\varrho_{2} \lambda .
$$

Furthermore, (3.4) is equivalent to the differential equations

$$
\begin{array}{ll}
\partial_{t}\left(\varrho_{1} v\right)+\operatorname{div}\left(\varrho_{1} v \otimes v+v \otimes J+\Pi^{1}\right)=\mathbf{f} & \text { in } \Omega^{1}, \\
\partial_{t}\left(\varrho_{2} v\right)+\operatorname{div}\left(\varrho_{2} v \otimes v+\Pi^{2}\right)=\mathbf{f} & \text { in } \Omega^{2},
\end{array}
$$

and, by using 3.8 , the continuity condition

$$
\left(\Pi^{1}-\Pi^{2}\right) v=0 \quad \text { on } \Gamma .
$$

To derive additional moving interface conditions we observe the following:

For $\lambda>0$ the characteristics of the hyperbolic equation 3.7 for $\varrho_{2}$ enter the interface. Therefore, the incoming $\varrho_{2}$-values have to be used in the boundary condition of the parabolic equation (3.7) for $\varrho_{1}$. For $\lambda<0$ the characteristics of (3.7) for $\varrho_{2}$ start at the interface, and therefore the values of $\varrho_{2}$ have to be prescribed.

This implies that a consistent interface condition for the values of $\left(\lambda, \varrho_{1}, \varrho_{2}\right) \in \mathbb{R}^{3}$ on $\Gamma$ consists of one equation for $\lambda>0$ and two equations for $\lambda<0$.

In order to derive such conditions we present a phase field model with a small parameter $\varepsilon$ describing the thickness of interfacial layers. Then an asymptotic expansion of such layers leads to an ODE system. From solvability considerations for this system we shall derive appropriate constitutive equations on the interface. They have the following form (for the proof of (3.13) see statement 6.5:

3.2. Constitutive equations. If, on the interface, $\left(\varrho_{1}, \varrho_{2}\right) \mapsto f_{d}\left(\varrho_{1}, \varrho_{2}\right)$ is defined as in equation 6.17), then the sign of $\lambda$ can be computed from the values of $\varrho_{1}$ and $\varrho_{2}$ by the formula

$$
\operatorname{sign} \lambda=\operatorname{sign} f_{d}\left(\varrho_{1}, \varrho_{2}\right) \text {. }
$$

Moreover, we obtain

$$
\begin{aligned}
\lambda & =\hat{\lambda}\left(\varrho_{1}, \varrho_{2}\right) & & \text { for } f_{d}\left(\varrho_{1}, \varrho_{2}\right)>0 \\
\varrho_{2} & =\hat{\varrho}_{2}\left(\varrho_{1}\right), \lambda=\hat{\lambda}\left(\varrho_{1}\right) & & \text { for } f_{d}\left(\varrho_{1}, \varrho_{2}\right)<0 .
\end{aligned}
$$

The identity (3.13) says that the sign of $\lambda$ is determined by the tangent of the graph of $f_{1}$ at $\varrho_{1}$ and the point $\left(\varrho_{2}, f_{2}\left(\varrho_{2}\right)\right.$ ) (see Fig. 5). If the point lies above the tangent, then $\lambda$ is positive, that is, the ordered phase shrinks. If it lies below the tangent, then $\lambda$ is negative, and the ordered phase grows. 


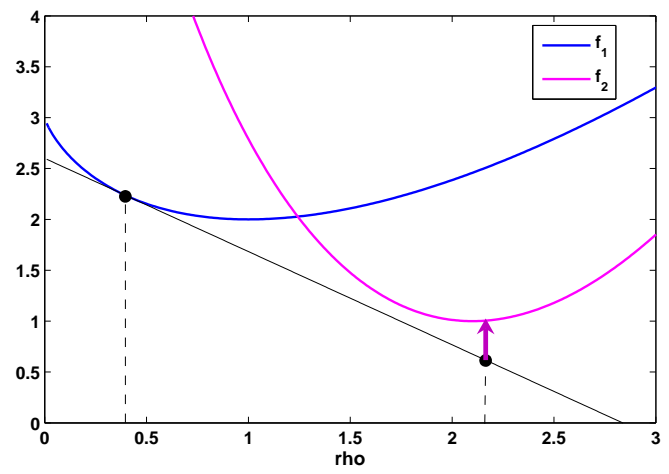

FIG. 5. Convex free energy functions $f_{1}$ and $f_{2}$ for disordered and ordered lipid phases, respectively, plotted over mixture density $\varrho$. Also drawn is the tangent of $f_{1}$ at the disordered phase density level $\varrho_{1}$. The propagation velocity $\lambda$ of a sharp interface transition from $\varrho_{1}$ to $\varrho_{2}$ has the same sign as the arrow between the tangent line and $f_{2}$ at the ordered phase density level $\varrho_{2}$.

\section{Generic phase field model}

We present a phase-field model for the interface problem of Section 3 based on the free energies $f_{1}$ and $f_{2}$ in 3.1 . We construct a free energy $f$ for the phase-field model depending on the gradient of the phase fraction. Moreover, we start with conservation laws for mass and momentum with a quite similar structure to that in 3.1. The main restrictions for this model are due to a free energy inequality (see [7], [1]). This inequality ensures that the model is consistent with thermodynamics. Independently, the phase field model could be used for numerical simulations. As mentioned above, as an outcome we shall obtain constitutive equations at the interface (see 3.14 ). They are used for numerical simulations of the interface problem.

In the phase-field model the densities $\varrho_{1}$ and $\varrho_{2}$ live in the entire domain. Total mass and phasefractions are defined by

$$
\varrho:=\varrho_{1}+\varrho_{2}, \quad \phi_{k}=\varrho_{k} / \varrho \quad \text { for } k=1,2, \quad \phi:=\phi_{2} .
$$

The general balance laws for the mass of each phase and the total momentum are

$$
\begin{aligned}
& \partial_{t} \varrho_{k}+\operatorname{div}\left(\varrho_{k} v+J_{k}\right)=r_{k}, \quad k=1,2, \\
& r_{1}+r_{2}=0, \\
& \partial_{t}(\varrho v)+\operatorname{div}\left(\varrho v \otimes v+v \otimes\left(J_{1}+J_{2}\right)+\Pi\right)=\mathbf{f} .
\end{aligned}
$$

Here $J_{k}$ are the diffusive mass fluxes and $r_{k}$ the mass transition rates. Moreover, $\Pi$ is the pressure tensor and $\mathbf{f}$ an external force. The specific forms of these quantities are described below, where the structure of $\Pi$ will be a consequence of the free energy inequality. Thus, so far the fluxes in (4.2) are arbitrary (the splitting into dynamical terms $\varrho_{k} v, \varrho v \otimes v$, and $v \otimes J_{k}$, which are well known from thermodynamics, is only done to simplify further computations). It follows that the total mass $\varrho$ satisfies

$$
\partial_{t} \varrho+\operatorname{div}(\varrho v+J)=0, \quad \text { where } J:=J_{1}+J_{2} \text {. }
$$


In general, we could allow the internal free energy $f$ depending on the densities and its spatial derivatives, that is,

$$
f=\tilde{f}\left(\varrho_{1}, \varrho_{2}, \nabla \varrho_{1}, \nabla \varrho_{2}\right)
$$

However, in a subdomain with $\varrho_{2} \approx 0$ (i.e. $\phi \approx 0$ ) we want $f$ to be approximately the internal free energy $\varrho_{1} \mapsto f_{1}\left(\varrho_{1}\right)$ in 3.1) of the pure disordered phase. Therefore, in this subdomain $f$ should be approximately independent of $\nabla \varrho_{1}$. The same considerations apply to regions with $\varrho_{1} \approx 0$ (i.e. $\phi \approx 1$ ). (We mention that this procedure is quite different from making a double-well potential out of $f_{1}$ and $f_{2}$, where certain parts of these free energies would be ignored.)

Assuming a symmetry at this stage, the only reasonable way to realize this is to allow $f$ to depend only on the gradient of $\phi$,

$$
\nabla \phi=\frac{\varrho_{1}}{\left(\varrho_{1}+\varrho_{2}\right)^{2}} \nabla \varrho_{2}-\frac{\varrho_{2}}{\left(\varrho_{1}+\varrho_{2}\right)^{2}} \nabla \varrho_{1}
$$

(Note that $\nabla \phi=\nabla \phi_{2}=-\nabla \phi_{1}$, since $\phi_{1}+\phi_{2}=1$.) Moreover, the dependence on $\left(\varrho_{1}, \varrho_{2}\right)$ is equivalent to a dependence on $(\varrho, \phi)$. Thus we arrive at the following form for the free energy:

4.1. Free energy. Consider a total free energy

$$
f_{\text {tot }}=f_{\text {tot }}(\varrho, \phi, v, \nabla \phi)=f_{\text {kin }}+f
$$

with kinetic free energy

$$
f_{\text {kin }}:=\frac{\varrho}{2}|v|^{2}
$$

and internal free energy

$$
f=f(\varrho, \phi, \nabla \phi) .
$$

The above splitting of $f_{\text {tot }}$ can be justified a posteriori by well known arguments from thermodynamics.

We shall consider solutions $\left(\varrho_{1}, \varrho_{2}, v\right)$ of (4.2) with certain constitutive relations for $J_{1}, J_{2}$, and $\Pi$, which are specified later (see 4.8 and 4.9 . We postulate:

4.2. Free energy inequality. With a free energy flux $\Psi_{\text {tot }}$ and constitutive equations, which have to be specified, the inequality

$$
\partial_{t} f_{\text {tot }}+\operatorname{div} \Psi_{\text {tot }} \leqslant v \bullet \mathbf{f}
$$

has to be satisfied for all solutions $\left(\varrho_{1}, \varrho_{2}, v\right)$ of system 4.2). We split

$$
\Psi_{\text {tot }}=f_{\text {tot }} v+\Pi^{\mathrm{T}} v+\frac{1}{2}|v|^{2} J+\Psi
$$

in well known kinetic terms and a vector $\Psi$.

4.3. Lemma. For the total free energy production we have

$$
g:=\partial_{t} f_{\text {tot }}+\operatorname{div} \Psi_{\text {tot }}-v \bullet \mathbf{f}=\partial_{t} f+\operatorname{div}(f v+\Psi)+D v \bullet \Pi .
$$

Thus 4.7) reads $g \leqslant 0$ for solutions of the system.

Proof. It is well known from fluid mechanics that for the kinetic terms

$$
\partial_{t} f_{\text {kin }}+\operatorname{div}\left(f_{\text {kin }} v+\Pi^{\mathrm{T}} v+\frac{1}{2}|v|^{2} J\right)=v \bullet \mathbf{f}+D v \bullet \Pi
$$

for solutions of 4.2 . 
Using the notation

$$
\dot{h}:=\partial_{t} h+v \bullet \nabla h \quad \text { for functions } h
$$

we have by the chain rule

$$
\partial_{t} f+\operatorname{div}(f v)=\dot{f}+f \operatorname{div} v=f^{\prime} \varrho \dot{\varrho}+f^{\prime} \phi \dot{\phi}+\sum_{i} f^{\prime}{ }_{\partial_{i} \phi} \partial_{i} \dot{\phi}+f \operatorname{div} v .
$$

From this we obtain

4.4. LEMMA. If

$$
\mu:=\frac{\delta f}{\delta \phi}=f_{\prime} \phi-\operatorname{div}\left(f^{\prime} \nabla \phi\right)
$$

denotes the first variation of $f$ with respect to $\phi$, then

$$
g=\operatorname{div}\left(\Psi+\dot{\phi} f^{\prime} \nabla \phi\right)+D v \bullet\left(f \operatorname{Id}-\nabla \phi \otimes f^{\prime} \nabla \phi+\Pi\right)+f^{\prime} \varrho \dot{\varrho}+\mu \dot{\phi} .
$$

Proof. Since

$$
\partial_{i} \dot{\phi}=\partial_{i} \dot{\phi}-\partial_{i} v \bullet \nabla \phi
$$

we obtain

$$
\begin{aligned}
& \sum_{i} f^{\prime} \partial_{i} \phi \partial_{i} \dot{\phi}=\sum_{i} f^{\prime} \partial_{i} \phi \partial_{i} \dot{\phi}-\sum_{i} f^{\prime} \partial_{i} \phi \partial_{i} v \bullet \nabla \phi \\
& =\sum_{i} \partial_{i}\left(f^{\prime} \partial_{i} \phi \dot{\phi}\right)-\left(\sum_{i} \partial_{i} f^{\prime} \partial_{i} \phi\right) \cdot \dot{\phi}-\sum_{i, j} \partial_{i} v_{j} f^{\prime} \partial_{i} \phi \partial_{j} \phi \\
& =\operatorname{div}\left(\dot{\phi} f^{\prime} \nabla \phi\right)-\dot{\phi} \operatorname{div} f^{\prime} \nabla \phi-D v \bullet\left(\nabla \phi \otimes f^{\prime} \nabla \phi\right) .
\end{aligned}
$$

Inserting this in (4.10) and then 4.10 into 4.8 yields the result.

Next we have to compute $\dot{\varrho}$ and $\dot{\phi}$ in 4.12.

4.5. Proposition. System 4.2 of differential equations is equivalent to the following for $(\varrho, v, \phi)$, where $\phi_{2}=\phi, \phi_{1}=1-\phi$, and $r:=r_{2}$ :

$$
\begin{aligned}
& \dot{\varrho}+\operatorname{div} J+\varrho \operatorname{div} v=0, \\
& \varrho \dot{\phi}+\phi_{1} \operatorname{div} J_{2}-\phi_{2} \operatorname{div} J_{1}=r, \\
& \varrho \dot{v}+\operatorname{div} \Pi+D v J=\mathbf{f} .
\end{aligned}
$$

Proof. The first identity follows from 4.3 and the last one from the momentum equation in 4.2. Moreover, from the second equation in 4.2 ,

$$
\begin{aligned}
r & =r_{2}=\partial_{t}(\phi \varrho)+\operatorname{div}\left(\phi \varrho v+J_{2}\right) \\
& =\varrho\left(\partial_{t} \phi+v \bullet \nabla \phi\right)+\phi\left(\partial_{t} \varrho+\operatorname{div}(\varrho v)\right)+\operatorname{div} J_{2} \\
& =\varrho \dot{\phi}-\phi \operatorname{div} J+\operatorname{div} J_{2}=\varrho \dot{\phi}+(1-\phi) \operatorname{div} J_{2}-\phi \operatorname{div} J_{1}
\end{aligned}
$$


Inserting this one obtains

$$
\begin{aligned}
f^{\prime} \varrho \dot{\varrho}+\mu \dot{\phi}= & -\varrho f^{\prime} \varrho \operatorname{div} v-f^{\prime} \varrho \operatorname{div} J+\frac{\mu}{\varrho}\left(r+\phi_{2} \operatorname{div} J_{2}-\phi_{2} \operatorname{div} J_{2}\right) \\
= & \operatorname{div}\left(\frac{\phi_{2} \mu}{\varrho} J_{1}-\frac{\phi_{1} \mu}{\varrho} J_{2}-f^{\prime} \varrho J\right)-\varrho f^{\prime} \varrho \operatorname{div} v+\frac{\mu}{\varrho} r \\
& +\nabla f^{\prime} \varrho \bullet J-\nabla\left(\frac{\phi_{2} \mu}{\varrho}\right) \bullet J_{1}+\nabla\left(\frac{\phi_{1} \mu}{\varrho}\right) \bullet J_{2},
\end{aligned}
$$

where $J=J_{1}+J_{2}$. Thus, using 4.12, we arrive at the final form for the free energy production: 4.6. Proposition. Define chemical potentials $\mu_{1}$ and $\mu_{2}$ and a pressure $p$ by

$$
\mu_{1}:=f^{\prime} \varrho-\frac{\phi_{2} \mu}{\varrho}, \quad \mu_{2}:=f^{\prime} \varrho+\frac{\phi_{1} \mu}{\varrho}, \quad p:=\varrho f^{\prime} \varrho-f .
$$

Then the free energy production $g$ becomes

$$
\begin{aligned}
g= & \operatorname{div}\left(\Psi+\dot{\phi} f^{\prime} \nabla \phi-\mu_{1} J_{1}-\mu_{2} J_{2}\right)+D v \bullet\left(-p \operatorname{Id}-\nabla \phi \otimes f^{\prime} \nabla \phi+\Pi\right) \\
& +\frac{\mu}{\varrho} r+\nabla \mu_{1} \bullet J_{1}+\nabla \mu_{2} \bullet J_{2} .
\end{aligned}
$$

4.7. REMARK. We have $\mu_{k}=\frac{\delta \tilde{f}}{\delta \varrho_{k}}$ for $k=1,2$, where $\tilde{f}$ is defined in 4.4.

Proof. Since $\phi=\varrho_{2} /\left(\varrho_{1}+\varrho_{2}\right)$, it follows that $\nabla \phi$ satisfies formula (4.5), so that

$$
\tilde{f}\left(\varrho_{1}, \varrho_{2}, \nabla \varrho_{1}, \nabla \varrho_{2}\right)=f\left(\varrho_{1}+\varrho_{2}, \frac{\varrho_{2}}{\varrho_{1}+\varrho_{2}}, \frac{\varrho_{1}}{\left(\varrho_{1}+\varrho_{2}\right)^{2}} \nabla \varrho_{2}-\frac{\varrho_{2}}{\left(\varrho_{1}+\varrho_{2}\right)^{2}} \nabla \varrho_{1}\right) .
$$

From this identity one derives the result.

We want the production rate $g$ to be nonpositive. The first term in (4.13) can be used to choose a particular free energy production $\Psi$, and the second by specifying a particular pressure tensor $\Pi$. The third term is dissipative.

4.8. ThEOREM. Assume that the free energy flux is given by

$$
\Psi:=-\dot{\phi} f^{\prime} \nabla \phi+\mu_{1} J_{1}+\mu_{2} J_{2}
$$

and let

$$
\Pi=p \operatorname{Id}+\nabla \phi \otimes f^{\prime} \nabla \phi-S .
$$

Then the free energy production is

$$
g=-D v \bullet S+\frac{\mu}{\varrho} r+\nabla \mu_{1} \bullet J_{1}+\nabla \mu_{2} \bullet J_{2} .
$$

Moreover, the free energy inequality is satisfied if

$$
\begin{gathered}
D v \bullet S \geqslant 0, \\
\frac{\mu}{\varrho} r+\sum_{k=1,2} \nabla \mu_{k} \bullet J_{k} \leqslant 0 .
\end{gathered}
$$


The term $-\dot{\phi} f^{\prime} \nabla \phi$ in the free energy flux is well known for phase field models, and the terms $\mu_{k} J_{k}$ occur in diffusion systems. The representation of $\Pi$ is analogous to models for a single fluid (the $\nabla \varrho$-terms in the Navier-Stokes-Korteweg equation), here the gradient terms involve only $\nabla \phi$.

The standard example satisfying (4.14) is the usual stress tensor $S=\alpha(D v)^{\mathrm{S}}+\beta \operatorname{div} v \operatorname{Id}$ for viscous fluids, where the Lamé coefficients are allowed to depend on $\varrho$ and $\phi$ with $\alpha>0$ and $(1 / n) \alpha+\beta>0$. Thus it remains to study inequality 4.15 .

The first term in (4.15) has the usual structure as for a reaction equation, and the second term is common for diffusion systems. Therefore, to ensure that $(4.15)$ holds, one could try to satisfy the two inequalities $(\mu / \varrho) r \leqslant 0$ and $\sum_{k=1,2} \nabla \mu_{k} \bullet J_{k} \leqslant 0$. However, in our case both terms are strongly coupled due to the form of the chemical potentials $\mu_{1}$ and $\mu_{2}$. Using this structure we obtain

4.9. Proposition. Define $K:=\phi_{1} J_{2}-\phi_{2} J_{1}$. Then with $J=J_{1}+J_{2}$ we obtain

$$
J_{1}=\phi_{1} J-K, \quad J_{2}=\phi_{2} J+K,
$$

and inequality 4.15 is equivalent to

$$
\frac{\mu}{\varrho}(r-\nabla \phi \bullet J)+\nabla f^{\prime} \varrho \bullet J+\nabla\left(\frac{\mu}{\varrho}\right) \bullet K \leqslant 0 .
$$

Proof. Using the definition of $\mu_{1}$ and $\mu_{2}$ in 4.6 with $\mu$ defined in (4.11) one gets

$$
\begin{aligned}
\nabla \mu_{1} \bullet J_{1}+\nabla \mu_{2} \bullet J_{2} & =\left(\phi_{1} \nabla \mu_{1}+\phi_{2} \nabla \mu_{2}\right) \bullet J+\nabla\left(\mu_{2}-\mu_{1}\right) \bullet K \\
& =\left(\phi_{1} \nabla\left(f^{\prime} \varrho-\frac{\phi_{2}}{\varrho} \mu\right)+\phi_{2} \nabla\left(f^{\prime} \varrho+\frac{\phi_{1}}{\varrho} \mu\right)\right) \bullet J+\nabla\left(\frac{\mu}{\varrho}\right) \bullet K
\end{aligned}
$$

and

$$
\begin{aligned}
& \phi_{1} \nabla\left(f^{\prime} \varrho-\frac{\phi_{2}}{\varrho} \mu\right)+\phi_{2} \nabla\left(f^{\prime} \varrho+\frac{\phi_{1}}{\varrho} \mu\right)=\nabla f^{\prime} \varrho-\frac{\phi_{1} \mu}{\varrho} \nabla \phi_{2}+\frac{\phi_{2} \mu}{\varrho} \nabla \phi_{1} \\
& =\nabla f^{\prime} \varrho-\left(\frac{\phi_{1} \mu}{\varrho}+\frac{\phi_{2} \mu}{\varrho}\right) \nabla \phi=\nabla f_{f^{\prime} \varrho}-\frac{\mu}{\varrho} \nabla \phi .
\end{aligned}
$$

Now, we can treat the terms in 4.16 as independent terms. The simplest case for $K$, provided $K$ is nontrivial, is to be a negative multiple of $\nabla(\mu / \varrho)$. Since $\mu$, by [4.11), is a second order operator in $\phi$, this would imply that the mass equations (in Proposition 4.5) would be of fourth order in $\phi$ (as for the Cahn-Hilliard equation). Thus, only the case $K=0$ leads to second order equations, which is the model we consider in this paper. Assuming $K=0$ means that the diffusive fluxes $J_{k}=\phi_{k} J$ are just the phase fractions of one common flux $J$.

4.10. General system. Assume $K=0$. Then the system of conservation laws (4.2) to solve is

$$
\begin{aligned}
& \partial_{t} \varrho+\operatorname{div}(\varrho v+J)=0, \\
& \partial_{t}(\phi \varrho)+\operatorname{div}(\phi \varrho v+\phi J)=r, \\
& \partial_{t}(\varrho v)+\operatorname{div}(\varrho v \otimes v+v \otimes J+\Pi)=\mathbf{f}
\end{aligned}
$$

with $r=r_{2}$ and $\Pi=p \operatorname{Id}+\nabla \phi \otimes f^{\prime} \nabla \phi-S$. This system is equivalent to (using the notation in (4.9)

$$
\begin{aligned}
& \dot{\varrho}+\varrho \operatorname{div} v+\operatorname{div} J=0, \\
& \varrho \dot{\phi}=r-\nabla \phi \bullet J, \\
& \varrho \dot{v}+\operatorname{div}\left(p \operatorname{Id}+\nabla \phi \otimes f^{\prime} \nabla \phi-S\right)+D v J=\mathbf{f} .
\end{aligned}
$$


In order to satisfy the free energy inequality, we are left with the first two terms in (4.16). The second term will be nonpositive, if we choose $J \sim-\nabla f^{\prime} \varrho$. Assuming $f=f_{0}(\varrho, \phi)+\frac{\varepsilon^{2}}{2}|\nabla \phi|^{2}$ the $\varrho$-equation in 4.17) will become a diffusion equation for $\varrho$. And for the sign of the first term we assume that $r-\nabla \phi \bullet J \sim-\mu$. Then the $\phi$-equation in (4.17) will be an Allen-Cahn type equation. Notice that then the transition rate $r$ contains a contribution from the diffusion flux $J$, leading to the fact that this term does no longer appear in the $\phi$-equation. Altogether we obtain

4.11. Special model. Let

$$
\begin{gathered}
f(\varrho, \phi, \nabla \phi)=f_{0}(\varrho, \phi)+\frac{\varepsilon^{2}}{2}|\nabla \phi|^{2}, \\
J=-d_{\varepsilon} \nabla\left(f_{0^{\prime} \varrho}\right), \quad d_{\varepsilon}=d_{\varepsilon}(\varrho, \phi)>0, \\
S=\alpha(\varrho, \phi)(D v)^{\mathrm{S}}+\beta(\varrho, \phi) \operatorname{div}(v) \mathrm{Id}, \quad \alpha>0, \frac{1}{n} \alpha+\beta \geqslant 0, \\
r=\nabla \phi \bullet J-\varrho \cdot c_{\varepsilon} \mu, \quad c_{\varepsilon}=c_{\varepsilon}(\varrho, \phi)>0,
\end{gathered}
$$

where the chemical potential is $\mu=\delta f / \delta \phi=f_{0^{\prime} \phi}-\varepsilon^{2} \Delta \phi$. Then the free energy inequality is satisfied and system (4.17) becomes

$$
\begin{aligned}
& \dot{\varrho}+\varrho \operatorname{div} v-\operatorname{div}\left(d_{\varepsilon} \nabla f_{0^{\prime} \varrho}\right)=0, \\
& \dot{\phi}+c_{\varepsilon} \cdot\left(f_{0^{\prime} \phi}-\varepsilon^{2} \Delta \phi\right)=0, \\
& \varrho \dot{v}+\operatorname{div}\left(P_{\varepsilon}-\alpha(D v)^{\mathrm{S}}-\beta \operatorname{div}(v) \mathrm{Id}\right)-d_{\varepsilon} D v \nabla f_{0^{\prime} \varrho}=\mathbf{f},
\end{aligned}
$$

where

$$
P_{\varepsilon}:=p_{0} \mathrm{Id}+\varepsilon^{2}\left(\nabla \phi \otimes \nabla \phi-\frac{1}{2}|\nabla \phi|^{2} \mathrm{Id}\right)
$$

with the classical pressure $p_{0}:=\varrho f_{0} \varrho-f_{0}$.

\section{Asymptotic expansion}

Let $\Omega^{1}$ and $\Omega^{2}$ denote two local domains in $\mathbb{R} \times \mathbb{R}^{n}$ separated by an evolving surface $\Gamma$. For each time $t$ we let

$$
\Gamma_{t}:=\{x:(t, x) \in \Gamma\}, \quad \Omega_{t}^{1}:=\left\{x:(t, x) \in \Omega^{1}\right\}, \quad \text { etc. }
$$

We deal with a family of solutions of PDE's with a small parameter $\varepsilon$, where $\varepsilon$ corresponds to an interfacial layer around $\Gamma$ of thickness $\mathcal{O}(\varepsilon)$. If $\phi$ is an order parameter in a phase field model, this means that for all fixed $\delta>0$ and all $t$ the set $\{x: \delta \leqslant \phi(t, x) \leqslant 1-\delta\}$ lies in an $\mathcal{O}(\varepsilon)$ neighbourhood of $\Gamma_{t}$. For a quantity $u$ we write

$$
\begin{aligned}
& u(t, x)=U\left(t, y(t, x), \frac{1}{\varepsilon} z(t, x)\right), \\
& U: \Gamma \times \mathbb{R} \rightarrow \mathbb{R}, \quad(t, y, \xi) \mapsto U(t, y, \xi), \\
& z(t, x) \text { signed distance of } x \text { from } \Gamma_{t}, z<0 \text { in } \Omega^{1}, z>0 \text { in } \Omega^{2}, \\
& y(t, x) \in \Gamma_{t} \text { projection of } x \text { on } \Gamma_{t} .
\end{aligned}
$$


The inner expansion of $u$ is of the form $U=U^{0}+\varepsilon U^{1}+\cdots$. The following formulas hold (with $y=y(t, x))$ :

$$
\begin{aligned}
& \nabla z(t, x)=v_{\Gamma}(t, y), \quad v_{\Gamma}(t, \bullet) \text { outer normal of } \Omega_{t}^{1} \\
& \partial_{t} y(t, x)=v_{\Gamma}(t, y), \quad v_{\Gamma}(t, \bullet) \text { velocity vector of } \Gamma_{t}, \\
& \partial_{t} z(t, x)=-v_{\Gamma}(t, y) \bullet v_{\Gamma}(t, y)+\mathcal{O}(\varepsilon), \\
& \Delta z(t, x)=-\kappa_{\Gamma}(t, y) \bullet v_{\Gamma}(t, y)+\mathcal{O}(\varepsilon), \quad \kappa_{\Gamma}(t, \bullet) \text { curvature vector of } \Gamma_{t}, \\
& \partial_{t} u=-\frac{1}{\varepsilon} \partial_{\xi} U v_{\Gamma} \bullet v_{\Gamma}+\partial_{t}^{\Gamma} U+\mathcal{O}(\varepsilon), \\
& \nabla u=\frac{1}{\varepsilon} \partial_{\xi} U v_{\Gamma}+\nabla_{y}^{\Gamma} U+\mathcal{O}(\varepsilon), \quad \operatorname{div} w=\frac{1}{\varepsilon} v_{\Gamma} \bullet \partial_{\xi} W+\mathcal{O}(1) \\
& \operatorname{div}(d \nabla u)=\frac{1}{\varepsilon^{2}} \partial_{\xi}\left(D \partial_{\xi} U\right)-\frac{1}{\varepsilon} D \kappa_{\Gamma} \bullet v_{\Gamma} \partial_{\xi} U+\mathcal{O}(1)
\end{aligned}
$$

In our case the quantities under consideration are

$$
\varrho=R\left(t, y, \frac{1}{\varepsilon} z\right), \quad \phi=\Phi\left(t, y, \frac{1}{\varepsilon} z\right), \quad v=V\left(t, y, \frac{1}{\varepsilon} z\right) .
$$

We take the equations in 4.18 of the special model and set

$$
c_{\varepsilon}(\varrho, \phi)=\frac{1}{\varepsilon} c(\varrho, \phi) \quad(\text { later } c=1)
$$

(for the usual Allen-Cahn equation we have $c_{\varepsilon}=c / \varepsilon^{2}$, a term which does not occur here). The diffusion coefficient $d_{\varepsilon}$ is supposed to satisfy the universal interpolation formula (in accordance with the theory of homogenization)

$$
\frac{1}{d_{\varepsilon}(\varrho, \phi)}=\frac{1}{\varepsilon d(\varrho)} h(\phi)+\frac{1}{d(\varrho)}(1-h(\phi)),
$$

with a continuous monotone function $h:[0,1] \rightarrow \mathbb{R}, h(0)=0, h(1)=1$. It follows that $d_{\varepsilon}(\varrho, 0)=$ $d(\varrho)$ and $d_{\varepsilon}(\varrho, 1)=\varepsilon d(\varrho)$. Therefore the diffusion coefficient is $d(\varrho)$ in the 'disordered phase' and $\mathcal{O}(\varepsilon)$ in the 'ordered phase'. The above form of $d_{\varepsilon}$ implies

$$
\frac{d_{\varepsilon}}{\varepsilon}=\frac{d(\varrho)}{h(\phi)+\varepsilon(1-h(\phi))},
$$

which is important for the asymptotic expansion, where the condition $\phi^{*}=0$ plays an extra role. Here $\phi^{*}:=\inf \{\phi: h(\phi)>0\}$.

With these hypotheses, using the notation in 4.97,

$$
\dot{\phi}+\frac{c}{\varepsilon} \cdot\left(f_{0 \prime}^{\prime} \phi(\varrho, \phi)-\varepsilon^{2} \Delta \phi\right)=0
$$

becomes

$$
-\frac{1}{\varepsilon}\left(v_{\Gamma}-V\right) \bullet v_{\Gamma} \partial_{\xi} \Phi+\frac{c(R, \Phi)}{\varepsilon}\left(f_{0{ }^{\prime} \phi}(R, \Phi)-\partial_{\xi}^{2} \Phi\right)=\mathcal{O}(1) .
$$


Conservation of total mass

$$
\dot{\varrho}+\varrho \operatorname{div} v-\operatorname{div}\left(d_{\varepsilon} \nabla f_{0^{\prime} \varrho}\right)=0
$$

becomes

$$
\begin{aligned}
-\frac{1}{\varepsilon}\left(v_{\Gamma}-V\right) \bullet v_{\Gamma} \partial_{\xi} & R+\frac{1}{\varepsilon} R v_{\Gamma} \bullet \partial_{\xi} V \\
& -\frac{1}{\varepsilon} \partial_{\xi}\left(\frac{d_{\varepsilon}}{\varepsilon} \partial_{\xi} f_{0 \prime} \varrho(R, \Phi)\right)+\frac{d_{\varepsilon}}{\varepsilon} \kappa_{\Gamma} \bullet v_{\Gamma} \partial_{\xi} f_{0 \prime} \varrho^{\prime}(R, \Phi)=\mathcal{O}(1) .
\end{aligned}
$$

Conservation of momentum

$$
\varrho \dot{v}+\operatorname{div}\left(P_{\varepsilon}-\alpha D v^{\mathrm{S}}-\beta \operatorname{div} v \mathrm{Id}\right)-d_{\varepsilon} D v \nabla f_{0 \prime}^{\prime} \varrho=\mathbf{f}
$$

becomes, as $\varepsilon \rightarrow 0$,

$$
\begin{aligned}
\frac{1}{\varepsilon^{2}} \partial_{\xi}\left(\frac{\alpha(R, \Phi)}{2} \partial_{\xi} V+\left(\frac{\alpha(R, \Phi)}{2}+\beta(R, \Phi)\right) \partial_{\xi} V \bullet v_{\Gamma} v_{\Gamma}\right) \\
+\frac{d_{\varepsilon}}{\varepsilon^{2}} \partial_{\xi} f_{0^{\prime} \varrho}(R, \Phi) \partial_{\xi} V=\mathcal{O}\left(\frac{1}{\varepsilon}\right)
\end{aligned}
$$

Now we let

$$
\Phi=\Phi^{0}+\varepsilon \Phi^{1}+\cdots, \quad R=R^{0}+\varepsilon R^{1}+\cdots, \quad V=V^{0}+\varepsilon V^{1}+\cdots,
$$

and obtain the following

5.1. RESUlt. Assume for simplicity $c=1$, and look for a continuous triple $\left(\Phi^{0}, R^{0}, V^{0}\right)$ such that $\Phi^{0}$ is an increasing solution. Then the equation for $V^{0}$ implies that $V^{0}$ is constant in $\xi$ (in particular $v_{1}^{0}=v_{2}^{0}$ ), hence also (compare (3.9))

$$
\lambda:=\left(v_{\Gamma}-V^{0}\right) \bullet v_{\Gamma}
$$

is constant in $\xi$. The equations for $\Phi^{0}$ and $R^{0}$ become

$$
\begin{aligned}
& \partial_{\xi}^{2} \Phi^{0}+\lambda \partial_{\xi} \Phi^{0}=f_{0{ }^{\prime} \phi}\left(R^{0}, \Phi^{0}\right) \quad \text { in } \mathbb{R}, \\
& \partial_{\xi}\left(\frac{d\left(R^{0}\right)}{h\left(\Phi^{0}\right)} \partial_{\xi} f_{0{ }^{\prime} \varrho}\left(R^{0}, \Phi^{0}\right)+\lambda R^{0}\right)=0 \quad \text { in }\left\{\Phi^{0}>\phi^{*}\right\}, \\
& f_{0{ }^{\prime} \varrho}\left(R^{0}, \Phi^{0}\right)=f_{0}{ }^{\prime} \varrho\left(\varrho_{1}^{0}, 0\right) \quad \text { in }\left\{\Phi^{0}<\phi^{*}\right\},
\end{aligned}
$$

with the boundary conditions

$$
\begin{array}{ll}
\Phi^{0}(-\infty)=0, & \Phi^{0}(+\infty)=1, \\
R^{0}(-\infty)=\varrho_{1}^{0}, & R^{0}(+\infty)=\varrho_{2}^{0} \\
V^{0}(-\infty)=v_{1}^{0}, & V^{0}(+\infty)=v_{2}^{0}
\end{array}
$$


Proof. First we compute the $1 / \varepsilon$-term of (5.4) to obtain

$$
-\left(v_{\Gamma}-V^{0}\right) \bullet v_{\Gamma} \partial_{\xi} \Phi^{0}+c\left(R^{0}, \Phi^{0}\right)\left(f_{0^{\prime} \phi}\left(R^{0}, \Phi^{0}\right)-\partial_{\xi}^{2} \Phi^{0}\right)=0
$$

on the entire $\xi$-axis. Next we look at equation 5.5 . In $\{h(\Phi)=0\}$ locally the $1 / \varepsilon^{2}$-term is

$$
-\partial_{\xi}\left(d\left(R^{0}\right) \partial_{\xi} f_{0} \varrho^{\prime}\left(R^{0}, \Phi^{0}\right)\right)=0 .
$$

Since the interior of $\left\{h\left(\Phi^{0}\right)=0\right\}$ is $\left\{\Phi^{0}<\phi^{*}\right\}$ and it is assumed that $\Phi^{0}$ is monotone, it follows that the set $\left\{\Phi^{0}<\phi^{*}\right\}$ is a left interval. Therefore $\partial_{\xi} f_{0}{ }^{\prime} \varrho\left(R^{0}, \Phi^{0}\right)=\mathrm{const} / d\left(R^{0}\right)$ (here constant means a function independent of $\xi)$. Now $\left(R^{0}(\xi), \Phi^{0}(\xi)\right) \rightarrow\left(\varrho_{1}^{0}, 0\right)$ as $\xi \rightarrow-\infty$, hence $f_{0} \varrho\left(R^{0}(\xi), \Phi^{0}(\xi)\right) \rightarrow f_{0} \varrho^{\prime}\left(\varrho_{1}^{0}, 0\right)$. From this it follows that const $=0$, and therefore

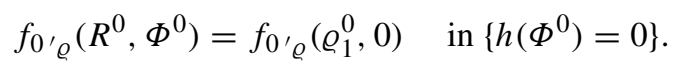

Locally in $\{h(\Phi)>0\}$ the $1 / \varepsilon$-term becomes

$$
\begin{aligned}
0 & =-\left(v_{\Gamma}-V^{0}\right) \bullet v_{\Gamma} \partial_{\xi} R^{0}+R^{0} v_{\Gamma} \bullet \partial_{\xi} V^{0}-\partial_{\xi}\left(\frac{d\left(R^{0}\right)}{h\left(\Phi^{0}\right)} \partial_{\xi} f_{0} \varrho\left(R^{0}, \Phi^{0}\right)\right) \\
& =-\partial_{\xi}\left(\frac{d\left(R^{0}\right)}{h\left(\Phi^{0}\right)} \partial_{\xi} f_{0^{\prime} \varrho}\left(R^{0}, \Phi^{0}\right)+\left(v_{\Gamma}-V^{0}\right) \bullet v_{\Gamma} R^{0}\right)
\end{aligned}
$$

hence

$$
\frac{d\left(R^{0}\right)}{h\left(\Phi^{0}\right)} \partial_{\xi} f_{0^{\prime} \varrho}\left(R^{0}, \Phi^{0}\right)+\left(v_{\Gamma}-V^{0}\right) \bullet v_{\Gamma} R^{0}=\text { const. }
$$

This implies that $\frac{d\left(R^{0}\right)}{h\left(\Phi^{0}\right)} \partial_{\xi} f_{0}{ }^{\prime}\left(R^{0}, \Phi^{0}\right)$ is a bounded function in $\left\{h\left(\Phi^{0}\right)>0\right\}$. Now let us look at 5.6. It follows that $d_{\varepsilon}(R, \Phi) \partial_{\xi} f_{0}{ }^{\prime} \varrho(R, \Phi)$ converges to 0 locally in $\{h(\Phi)>0\}$, since $d_{\varepsilon}(R, \Phi)=$ $\mathcal{O}(\varepsilon)$, and to 0 locally in $\{h(\Phi)=0\}$, since $\partial_{\xi} f_{0}{ }^{\prime}(R, \Phi) \rightarrow 0$. This shows that the $1 / \varepsilon^{2}$-term in (5.6) becomes

$$
\partial_{\xi}\left(\frac{\alpha\left(R^{0}, \Phi^{0}\right)}{2} \partial_{\xi} V^{0}+\left(\frac{\alpha\left(R^{0}, \Phi^{0}\right)}{2}+\beta\left(R^{0}, \Phi^{0}\right)\right) \partial_{\xi} V^{0} \bullet v_{\Gamma} v_{\Gamma}\right)=0
$$

in $\left\{\Phi^{0} \neq \phi^{*}\right\}$. Since $V^{0}(\xi) \rightarrow v_{2}^{0}$ for $\xi \rightarrow+\infty$ and $V^{0}(\xi) \rightarrow v_{1}^{0}$ for $\xi \rightarrow-\infty$, we conclude that $\partial_{\xi} V^{0}=0$ in $\left\{\Phi^{0} \neq \phi^{*}\right\}$. Hence, since $V^{0}$ is assumed to be continuous, it follows $v_{1}^{0}=V^{0}=v_{2}^{0}$.

Here $\lambda=\left(v_{\Gamma}-V^{0}\right) \bullet v_{\Gamma}=\left(v_{\Gamma}-v\right) \bullet v_{\Gamma}$ is the (relative) propagation speed of the interface (cf. (3.9)). $\lambda>0$ means that the ordered phase dissolves, and $\lambda<0$ means that the ordered phase grows.

We mention that the coefficient $d\left(R^{0}\right) / h\left(\Phi^{0}\right)$ is unbounded. If $\phi^{*}>0$ this happens at a finite point. In $\left\{\Phi^{0}<\phi^{*}\right\}$ the equation for the density $f_{0{ }^{\prime} \varrho}\left(R^{0}, \Phi^{0}\right)=f_{0^{\prime} \varrho}\left(\varrho_{1}^{0}, 0\right)$ gives $R^{0}$ in terms of

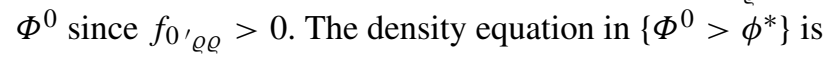

$$
\frac{d\left(R^{0}\right)}{h\left(\Phi^{0}\right)} \partial_{\xi} f_{0}{ }^{\varrho}\left(R^{0}, \Phi^{0}\right)+\lambda R^{0}=\text { const. }
$$


Letting $\xi \rightarrow+\infty$, it follows that the constant is $\lambda \varrho_{2}^{0}$. Therefore

$$
d\left(R^{0}\right) \partial_{\xi} f_{0} \varrho\left(R^{0}, \Phi^{0}\right)=-\lambda h\left(\Phi^{0}\right)\left(R^{0}-\varrho_{2}^{0}\right) \quad \text { in }\left\{\Phi^{0}>\phi^{*}\right\} .
$$

Thus the system to solve is

$$
\begin{aligned}
& \partial_{\xi}^{2} \Phi^{0}+\lambda \partial_{\xi} \Phi^{0}=f_{0^{\prime} \phi}\left(R^{0}, \Phi^{0}\right) \quad \text { in } \mathbb{R}, \\
& d\left(R^{0}\right) \partial_{\xi} f_{0^{\prime}} \varrho\left(R^{0}, \Phi^{0}\right)=-\lambda h\left(\Phi^{0}\right)\left(R^{0}-\varrho_{2}^{0}\right) \quad \text { in }\left\{\Phi^{0}>\phi^{*}\right\},
\end{aligned}
$$

and $f_{0^{\prime} \varrho}\left(R^{0}, \Phi^{0}\right)=f_{0^{\prime} \varrho}\left(\varrho_{1}^{0}, 0\right)$ in $\left\{\Phi^{0}<\phi^{*}\right\}$. Renaming $\Phi^{0} \leadsto \phi, R^{0} \leadsto \varrho, \partial_{\xi} \sim^{\prime}$, this is

$$
\begin{aligned}
& \phi^{\prime \prime}+\lambda \phi^{\prime}=f_{0^{\prime} \phi}(\varrho, \phi) \quad \text { in } \mathbb{R}, \\
& d(\varrho)\left(f_{0^{\prime}} \varrho(\varrho, \phi)\right)^{\prime}=-\lambda h(\phi)\left(\varrho-\varrho_{2}^{0}\right) \quad \text { in }\left\{\phi>\phi^{*}\right\},
\end{aligned}
$$

In the following sections we restrict to the case $\phi^{*}=0$.

5.2. Higher expansion. In order to show consistency with $(3.8)$ a further expansion is needed. For this the above estimate $\partial_{\xi} f_{0}{ }^{\prime} \varrho\left(R^{0}, \Phi^{0}\right)=\mathcal{O}\left(h\left(\Phi^{0}\right)\right)$ as $h\left(\Phi^{0}\right) \searrow 0$ is crucial for controlling the terms in the higher order expansion.

\section{Interfacial transition layer}

We solve the system [5.9) in the case $\phi^{*}=0$ and $h(\phi)=\phi$ :

$$
\begin{aligned}
& \phi^{\prime \prime}+\lambda \phi^{\prime}=f_{0{ }^{\prime} \phi}(\varrho, \phi), \\
& d(\varrho)\left(f_{0^{\prime} \varrho \varrho}(\varrho, \phi) \varrho^{\prime}+f_{0^{\prime} \varrho \phi}(\varrho, \phi) \phi^{\prime}\right)=-\lambda \phi\left(\varrho-\varrho_{2}^{0}\right),
\end{aligned}
$$

for $\phi, \varrho: \mathbb{R} \rightarrow \mathbb{R}$ with boundary conditions

$$
\begin{array}{ll}
\phi(-\infty)=0, & \phi(+\infty)=1, \\
\varrho(-\infty)=\varrho_{1}^{0}, & \varrho(+\infty)=\varrho_{2}^{0} .
\end{array}
$$

For solutions it follows that

$$
\phi^{\prime}(-\infty)=0, \quad \phi^{\prime}(+\infty)=0 .
$$

For the function $f_{0}(\phi, \varrho)$ defined in 4.11 we suppose the following:

6.1. Assumptions on the free energy. Assume that $\varrho \mapsto f_{1}(\varrho)$ and $\varrho \mapsto f_{2}(\varrho)$ are the given strictly convex free energies of the pure disordered and pure ordered phase in 3.1 . For $(\varrho, \phi) \mapsto f_{0}(\varrho, \phi)$ (introduced in 4.11) we assume that

$$
\begin{array}{ll}
f_{0}(\varrho, 0)=f_{1}(\varrho), & f_{0}(\varrho, 1)=f_{2}(\varrho), \\
f_{0^{\prime} \phi}(\varrho, 0)=0, & f_{0^{\prime} \phi}(\varrho, 1)=0, \\
f_{0^{\prime} \phi \phi}(\varrho, 0)>0, & f_{0^{\prime} \phi \phi}(\varrho, 1)>0 .
\end{array}
$$

It follows that $f_{0^{\prime} \varrho \phi}(\varrho, 0)=0$ and $f_{0^{\prime} \varrho \phi}(\varrho, 1)=0$.

Equation (6.4) says that $f_{0}$ is an interpolation between the given free energies $f_{1}$ and $f_{2}$ of the pure phases. Equation (6.5) means that this interpolation is not linear, and equation (6.6) implies 
that this interpolation is not monotone. There is a energy hump between the exact phases. This will be important for the mathematical treatment as well for the physical interpretation.

Most of the results in this section will be derived under these general assumptions. There are several possibilities to write down explicit formulas for $f_{0}$ satisfying the above conditions. Here we propose a simple approach to model nonlinear phase transition, where the defining terms are interpreted by underlying microscopic mechanisms:

6.2. Nonlinear free energy interpolation: Definition. Depending on the phase fraction parameter $0 \leqslant \phi \leqslant 1$ we choose a nonlinear phase separation mapping $\chi:[0,1] \rightarrow[0,1]$, which is bijective with $\chi^{\prime}(0)=\chi^{\prime}(1)=\chi^{\prime \prime}(0)=\chi^{\prime \prime}(1)=0, \chi^{\prime}(\phi)>0$ for $0<\phi<1$, and invariant under the symmetric exchange transformation $\phi \mapsto 1-\phi$ implying $\chi(1-\phi)=1-\chi(\phi)$. The simplest realization by an interpolating polynomial of $5^{\text {th }}$ order, which we choose here, gives $\chi^{\prime}(\phi)=30 \phi^{2}(1-\phi)^{2}$. For each phase we have, according to 4.1),

$$
\begin{array}{ll}
\phi_{1}=1-\phi, & \chi\left(\phi_{1}\right)=1-\chi(\phi), \\
\phi_{2}=\phi, & \chi\left(\phi_{2}\right)=\chi(\phi) .
\end{array}
$$

Here $\phi_{k}$ for $k=1,2$ denotes the relative amount of lipids in the mixture, so that $\chi\left(\phi_{k}\right)$ can be interpreted as the mean fraction of those lipids that have the chance for phase transition due to contact with lipids of the other phase. The s-shaped, threshold-like increase from $\chi(0)=0$ to $\chi(1 / 2)=1 / 2$ to $\chi(1)=1$ could be due to the local formation of microscopically small patches (e.g. micelles) reducing the free contact between lipids of the different phases. Under this condition we define the interpolated free energy at concentration $\varrho$ and phase parameter $\phi$ as

$$
f_{0}(\varrho, \phi)=\chi\left(\phi_{1}\right) \cdot F_{1}\left(\varrho, \phi_{1}\right)+\chi\left(\phi_{2}\right) \cdot F_{2}\left(\varrho, \phi_{2}\right),
$$

where for each phase $k=1,2$,

$$
F_{k}\left(\varrho, \phi_{k}\right)=f_{k}(\varrho)+\tilde{F}_{k}\left(\varrho, \tilde{\varrho}_{k}\left(\varrho, \phi_{k}\right)\right), \quad \tilde{\varrho}_{k}\left(\varrho, \phi_{k}\right)=\left(1-\theta_{k}\left(\phi_{k}\right)\right) \cdot \varrho
$$

with a positive function $\tilde{F}_{k}\left(\varrho, \tilde{\varrho}_{k}\right)$ denoting the extra free energy needed for phase transition out of phase $k$, and $0 \leqslant \theta_{k}\left(\phi_{k}\right) \leqslant 1$ the mean fraction of those contacting $k$-type lipids that, on average, perform such a transition by switching from phase $k$ to the other phase.

As a simple model for this to occur we consider first order saturation kinetics of Monod type:

$$
\theta_{k}\left(\phi_{k}\right)=\frac{s_{k} \phi_{k}}{1+s_{k} \phi_{k} / \vartheta_{k}}
$$

with scaling parameters $s_{k} \leqslant 1$ and saturation values $\vartheta_{k} \leqslant 1$. Before we specify a modeling definition of the transition energy $\tilde{F}_{k}$, let us look at the so-called excess free energy resulting as difference between $f_{0}$ and the standard linear interpolation energy:

$$
\begin{aligned}
f_{\mathrm{ex}}(\varrho, \phi) & =f_{0}(\varrho, \phi)-(1-\phi) f_{1}(\varrho)-\phi f_{2}(\varrho) \\
& =(\phi-\chi(\phi)) \cdot\left(f_{1}(\varrho)-f_{2}(\varrho)\right)+\chi\left(\phi_{1}\right) \cdot \tilde{F}_{k}\left(\varrho, \tilde{\varrho}_{1}\right)+\chi\left(\phi_{2}\right) \cdot \tilde{F}_{k}\left(\varrho, \tilde{\varrho}_{2}\right) .
\end{aligned}
$$

The product in the first term is, say for the case $\phi_{2}=\phi<1 / 2$, proportional to the amount $\phi-\chi(\phi)$ of those 2-type lipids that do not have the chance of phase transition (e.g. by being 
bound in micelles), thereby increasing (or decreasing) the effective free energy proportional to the experienced energy jump $f_{1}(\varrho)-f_{2}(\varrho)$. The last two terms in 6.11 measure the free energy of those $k$-type lipids having a chance of phase transition; if they undergo such a transformation, they contribute to the density interval $I_{k}=\left[\tilde{\varrho}_{k}, \varrho\right]$ above the density $\tilde{\varrho}_{k}=\left(1-\theta_{k}\left(\phi_{k}\right)\right) \varrho$ of remaining nontransformed lipids. A reasonable property of the extra transition energy $\tilde{F}_{k}\left(\varrho, \tilde{\varrho}_{k}\right)$ should be that an extension of the density $\operatorname{span}\left|I_{k}\right|=\varrho-\tilde{\varrho}_{k}=\theta_{k}\left(\phi_{k}\right) \varrho$ by lowering $\tilde{\varrho}_{k}$ induces an increase of free transition energy equal to the difference of the chemical potentials $\mu_{k}$ between the situation before and after phase transition. This gives the differential equation

$$
-\frac{\mathrm{d}}{\mathrm{d} \sigma} \tilde{F}_{k}(\varrho, \sigma)=\int_{\sigma}^{\varrho} f_{k}^{\prime \prime}(r) \mathrm{d} r=\mu_{k}(\varrho)-\mu_{k}(\sigma)>0 .
$$

Integration with respect to $\sigma$ then reveals the explicit model function

$$
\tilde{F}_{k}(\varrho, \sigma)=\int_{\sigma}^{\varrho} \int_{r}^{\varrho} f_{k}^{\prime \prime}(\tilde{r}) \mathrm{d} \tilde{r} \mathrm{~d} r=f_{k}(\sigma)-F_{k}^{\operatorname{lin}}(\varrho, \sigma)>0
$$

with $F_{k}^{\text {lin }}(\varrho, \sigma)=f_{k}(\varrho)+(\sigma-\varrho) f_{k}^{\prime}(\varrho)$ representing the tangent line at $\varrho$ below the convex free energy function $f_{k}$ (see also Fig. 5). Thus, the extra transition energy in (6.13) just measures the height of the relative free energy well of phase $k$ over its tangent line at the given concentration $\varrho$.

6.3. Standard example: Gibbs free energies. As a most simple prototypical example let us consider the standard convex Gibbs energies introduced in (2.2) and (2.4) with $a_{k}=0$ :

$$
f_{k}(\varrho)=b_{k} \cdot \varrho \cdot\left(\ln \frac{\varrho}{\varrho_{k}^{*}}-1\right)+\text { const. }
$$

Straightforward computation then yields transition energies 6.13 that are proportional to $\varrho$ with coefficients only depending on the transition fraction $\theta_{k}=\theta_{k}\left(\phi_{k}\right)$ in 6.10, namely

$$
\tilde{F}_{k}\left(\varrho,\left(1-\theta_{k}\right) \varrho\right)=b_{k} \cdot \beta\left(\theta_{k}\right) \cdot \varrho
$$

with the monotone function $\beta(\theta)=\theta+(1-\theta) \cdot \ln (1-\theta)$ satisfying $\beta(0)=\beta^{\prime}(0)=0$ and $\beta(1)=1$. Thus, the last two terms in the excess free energy (6.11) are just $\tilde{b}_{k}\left(\phi_{k}\right) \cdot \varrho$ with $\tilde{b}_{k}\left(\phi_{k}\right)=$ $b_{k} \chi\left(\phi_{k}\right) \beta\left(\theta_{k}\left(\phi_{k}\right)\right)$ for each phase $k=1,2$ and some positive parameters $s_{k} \leqslant 1$ and $\theta_{k} \leqslant 1$. The choice of these two parameters in 6.10 determines height and skewness of the appearing free energy hump, thus modeling the energetics of transitions from phase $k$ to the other phase; see the $\phi$-profile of $f_{\text {ex }}$ for the largest value $\varrho=4$ in Fig. 6a. The hump is always steeper at the own-type phase side $\left(\phi_{k}=1\right)$. On the other hand, for low values of $\varrho$, these humps in the excess free energy are diminished and dominated by the first term in (6.11), the sign changing function $(\phi-\chi(\phi)) \cdot\left(f_{1}(\varrho)-f_{2}(\varrho)\right)$. This is clearly visible in the plots of $f_{\text {ex }}$ and of the resulting interpolated free energy function $f_{0}$ in Fig. 6 .

Let us continue the consideration under the general assumptions 6.1. In the case without diffusion, that is, $d=0$, it follows from the second equation in 6.1 that $\varrho=\varrho_{2}^{0}$ (provided $\lambda \neq 0$ ), and therefore $\varrho_{1}^{0}=\varrho_{2}^{0}$. For such a continuous phase transition it remains to solve the first equation in 6.1. 

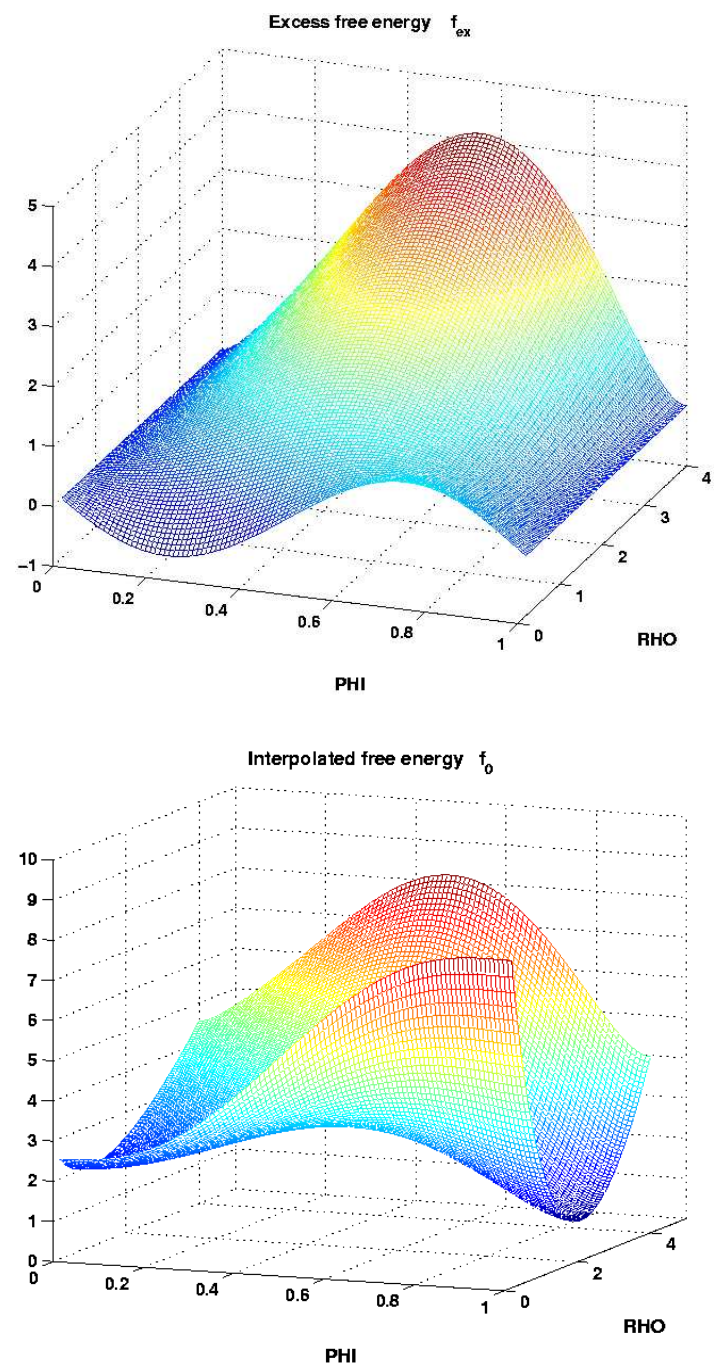

FIG. 6. Free energy obtained by nonlinear interpolation between two Gibbs energies with different steepness $\left(b_{1}=1\right.$, $\left.b_{2}=5\right)$ and minimum $\left(c_{1}=2, c_{2}=1\right)$ as in Fig. 44. Other parameters are $\vartheta_{k}=1, s_{1}=9$ and $s_{2}=4$. Plotted over $\phi$ and $\varrho$ are (a) (upper panel) the excess free energy $f_{\text {ex }}$ and (b) (lower panel) the full interpolated free energy $f_{0}$.

6.4. Case without diffusion. For given $\varrho=$ const consider the boundary value problem

$$
\phi^{\prime \prime}+\lambda \phi^{\prime}=f_{0^{\prime} \phi}(\varrho, \phi), \quad \phi(-\infty)=0, \phi(+\infty)=1,
$$

for $\phi: \mathbb{R} \rightarrow \mathbb{R}$ with parameter $\lambda \in \mathbb{R}$. Then for $f_{2}(\varrho) \neq f_{1}(\varrho)$ there exists a unique value $\hat{\lambda}(\varrho) \neq 0$ such that a solution $\phi$ exists if and only if the interface propagation speed is $\lambda=\hat{\lambda}(\varrho)$. Moreover, 
we obtain the following sign condition:

$$
\operatorname{sign} \lambda=\operatorname{sign}\left(f_{2}(\varrho)-f_{1}(\varrho)\right) .
$$

Proof. The statement about the sign follows from the energy method (as in the following proof). The scaling property is easy to see. The existence follows from standard phase diagram considerations using the assumptions in 6.1, in particular 6.6.

For the case with diffusion we generally have $\varrho_{2}^{0} \neq \varrho_{1}^{0}$ and obtain, by using the energy method:

6.5. Case with diffusion: Lemma. Define the indicator function (see Fig. 5 and Fig. 77)

$$
f_{d}\left(\varrho_{1}, \varrho_{2}\right):=f_{2}\left(\varrho_{2}\right)-\left(f_{1}\left(\varrho_{1}\right)+\left(\varrho_{2}-\varrho_{1}\right) f_{1}^{\prime}\left(\varrho_{1}\right)\right)
$$

for values $\varrho_{1}$ and $\varrho_{2}$. Then for solutions of 6.1 with 6.2) the value $f_{d}\left(\varrho_{1}^{0}, \varrho_{2}^{0}\right)$ determines the sign of the interface propagation speed:

$$
\operatorname{sign} \lambda=\operatorname{sign} f_{d}\left(\varrho_{1}^{0}, \varrho_{2}^{0}\right) .
$$
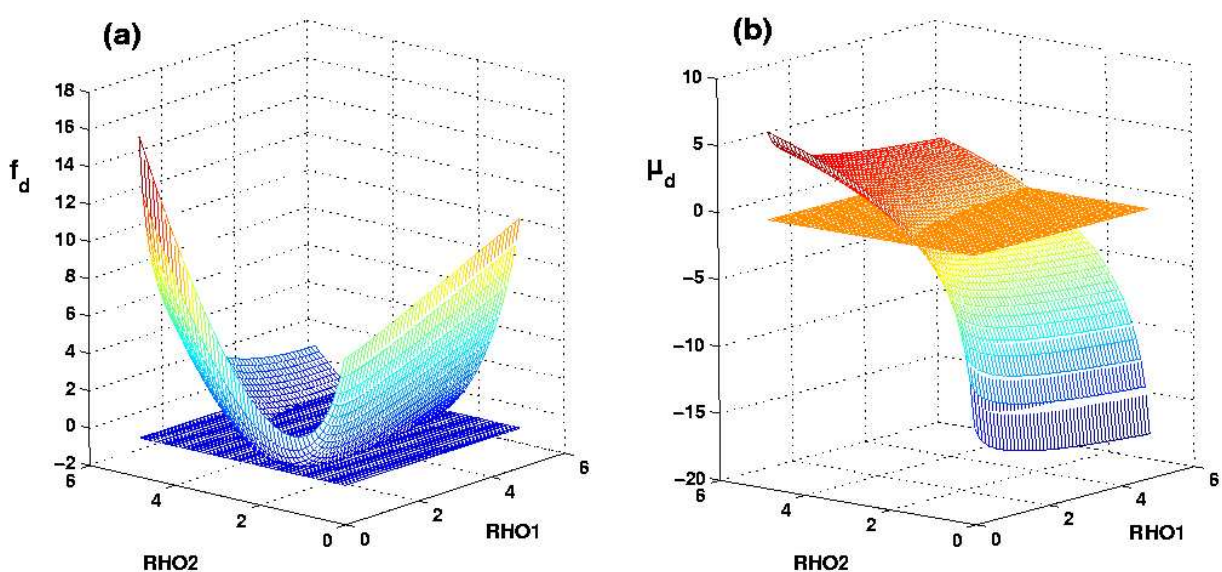

FIG. 7. Plot of the $\lambda$-sign indicator functions and the intersecting zero level planes: (a) $f_{d}\left(\varrho_{1}, \varrho_{2}\right)$ defined in 6.17) and (b) $\mu_{d}\left(\varrho_{1}, \varrho_{2}\right)$ defined in 6.18. Parameters as in Fig. 6

Remark. For $\varrho_{1}^{0}=\varrho_{2}^{0}$ this reduces to the identity 6.16.

Proof. For $c \in \mathbb{R}$ consider the relative pressure

$$
p_{c}(\varrho, \phi):=(\varrho-c) f_{0 \prime} \varrho(\varrho, \phi)-f_{0}(\varrho, \phi) .
$$

Then, by using the $\phi^{\prime}$-equation in 6.1,

$$
\begin{aligned}
& \left(\frac{1}{2}\left|\phi^{\prime}\right|^{2}+p_{c}(\varrho, \phi)\right)^{\prime}=\left(\phi^{\prime \prime}+p_{c^{\prime} \phi}\right) \phi^{\prime}+p_{c^{\prime} \varrho} \varrho^{\prime} \\
& =-\lambda\left|\phi^{\prime}\right|^{2}+(\varrho-c) f_{0}{ }^{\prime} \varrho \phi \cdot \phi^{\prime}+(\varrho-c) f_{0}{ }^{\prime} \varrho \varrho(\varrho, \phi) \varrho^{\prime}=-\lambda\left|\phi^{\prime}\right|^{2}+(\varrho-c)\left(f_{0 \prime}{ }^{\prime}\right)^{\prime} \\
& =-\lambda\left|\phi^{\prime}\right|^{2}-\frac{\lambda}{d(\varrho)} \phi(\varrho-c)\left(\varrho-\varrho_{2}^{0}\right)=-\lambda \cdot\left(\left|\phi^{\prime}\right|^{2}+\frac{\phi}{d(\varrho)}\left(\varrho-\varrho_{2}^{0}\right)^{2}\right)
\end{aligned}
$$


if we set $c=\varrho_{2}^{0}$. Then integration leads to

$$
\lambda \int_{\mathbb{R}}\left(\left|\phi^{\prime}\right|^{2}+\frac{\phi}{d(\varrho)}\left(\varrho-\varrho_{2}^{0}\right)^{2}\right) \mathrm{d} \xi=-\left[p_{c}(\varrho, \phi)\right]_{-\infty}^{+\infty},
$$

which with 6.2) gives the result, since the integral is positive.

Moreover, we show the following

6.6. LEMMA. Define the difference of chemical potentials between the two phases $k=1,2$ at given concentrations $\varrho_{k}$ as a second indicator function (see Fig. 7p)

$$
\mu_{d}\left(\varrho_{1}, \varrho_{2}\right):=f_{2}^{\prime}\left(\varrho_{2}\right)-f_{1}^{\prime}\left(\varrho_{1}\right)=\mu_{2}\left(\varrho_{2}\right)-\mu_{1}\left(\varrho_{1}\right)
$$

Then for solutions of system (6.1) with boundary condition 6.2 ,

$$
\mu_{d}\left(\varrho_{1}^{0}, \varrho_{2}^{0}\right)=\lambda \cdot \int_{\mathbb{R}} \frac{\phi}{d(\varrho)}\left(\varrho_{2}^{0}-\varrho\right) \mathrm{d} \xi
$$

Proof. Divide the second equation in 5.9) by $d(\varrho)$ and then integrate over $\mathbb{R}$. This gives

$$
\lambda \cdot \int_{\mathbb{R}} \frac{\phi}{d(\varrho)}\left(\varrho_{2}^{0}-\varrho\right) \mathrm{d} \xi=f_{0{ }^{\prime} \varrho}(\varrho, \phi)(-\infty)-f_{0{ }^{\prime} \varrho}(\varrho, \phi)(+\infty)=f_{0{ }^{\prime} \varrho}\left(\varrho_{1}^{0}, 0\right)-f_{0{ }^{\prime} \varrho}\left(\varrho_{2}^{0}, 1\right) .
$$

Now, by 6.4 , we know that $f_{0}(\varrho, 0)=f_{1}(\varrho)$ for every $\varrho$, therefore also $f_{0 \prime} \varrho(\varrho, 0)=f_{1}^{\prime}(\varrho)$, thus

$$
f_{0 \prime} \varrho\left(\varrho_{1}^{0}, 0\right)=f_{1}^{\prime}\left(\varrho_{1}^{0}\right)=\mu_{1}\left(\varrho_{1}^{0}\right)
$$

Inserting also the analogous equation for $f_{0}(\varrho, 1)=f_{2}(\varrho)$, we obtain the result.

Moreover, defining the difference of the pressures by

$$
p_{d}\left(\varrho_{1}, \varrho_{2}\right)=p_{2}\left(\varrho_{2}\right)-p_{1}\left(\varrho_{1}\right) \varrho_{2} \cdot \mu_{d}\left(\varrho_{1}, \varrho_{2}\right)-f_{d}\left(\varrho_{1}, \varrho_{2}\right),
$$

we deduce the following lemma.

6.7. Case $\lambda=0$ : Lemma. Solutions of (6.1) and 6.2 with zero interface speed $\lambda=0$ have to fulfill both conditions $f_{d}\left(\varrho_{1}^{0}, \varrho_{2}^{0}\right)=0$ and $\mu_{d}\left(\varrho_{1}^{0}, \varrho_{2}^{0}\right)=0$. This is equivalent to the fact that the difference of the chemical potentials and the difference of the pressures in the two phases vanish.

Remark. The ordinary differential equations boil down to the single equation $\phi^{\prime \prime}=f_{0^{\prime} \phi}(\hat{\varrho}(\phi), \phi)$. Special case. Thus, if the curves intersect transversally as in the example, the appearing cross point $\left(\varrho_{1}^{\text {sing }}, \varrho_{2}^{\text {sing }}\right)$ defines the (locally) unique transition solution of 6.1 ) and 6.2) satisfying $\lambda=0$. (See the plot of the zero level curves in Fig. 8.)

Proof. We have $f_{d}\left(\varrho_{1}^{0}, \varrho_{2}^{0}\right)=0$ by 6.5 and $\mu_{d}\left(\varrho_{1}^{0}, \varrho_{2}^{0}\right)=0$ by 6.6 By 6.20) this is equivalent to $p_{d}\left(\varrho_{1}^{0}, \varrho_{2}^{0}\right)=0$ and $f_{d}\left(\varrho_{1}^{0}, \varrho_{2}^{0}\right)=0$. The second equation in 5.9 leads to $f_{0}{ }^{\prime} \varrho(\varrho, \phi)=$ const, hence as in the previous proof

$$
f_{0 \prime} \varrho(\varrho, \phi)=f_{1}^{\prime}\left(\varrho_{1}^{0}\right)=f_{2}^{\prime}\left(\varrho_{2}^{0}\right) .
$$

Since $f_{0} \varrho \varrho>0$ there is exactly one curve $\varrho=\hat{\varrho}(\phi)$ satisfying this identity. This implies the remark. 


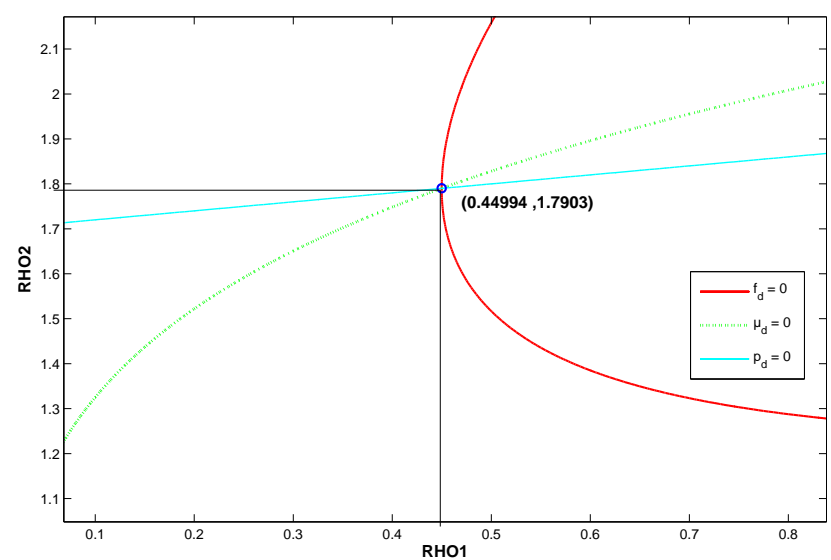

FIG. 8. Zoomed plot of the zero level curves for the sign indicator functions $f_{d}$ (Fig. $7 \mathrm{k}$ ), $\mu_{d}$ (Fig. $7 \mathrm{p}$ ), and $p_{d}$ defined in 6.20. The singular point $\varrho_{1}^{\text {sing }} \approx 0.45, \varrho_{2}^{\text {sing }} \approx 1.79$ is the intersection of all three level curves.

System 6.1 is a first order system for $\left(\phi, \phi^{\prime}, \varrho\right)$ in $\mathbb{R}^{3}$, for which a general existence result is not available. To study solvability, we linearize the system at $\left(0,0, \varrho_{1}^{0}\right)$ and $\left(1,0, \varrho_{2}^{0}\right)$. This leads to the matrices

$$
\left[\begin{array}{ccc}
0 & 1 & 0 \\
f_{0 \prime}^{\prime} \phi \phi & -\lambda & 0 \\
-\frac{\lambda\left(\varrho_{1}^{0}-\varrho_{2}^{0}\right)}{f_{0^{\prime} \varrho e^{d}}} & 0 & 0
\end{array}\right] \text { and }\left[\begin{array}{ccc}
0 & 1 & 0 \\
f_{0^{\prime} \phi \phi} & -\lambda & 0 \\
0 & 0 & -\frac{\lambda}{f_{0^{\prime} \varrho e^{d}}}
\end{array}\right] \text {, }
$$

where in the first matrix $f_{0^{\prime} \phi \phi}=f_{0{ }^{\prime} \phi \phi}\left(\varrho_{1}^{0}, 0\right)>0, f_{0^{\prime} \varrho \varrho}=f_{1}^{\prime \prime}\left(\varrho_{1}^{0}\right)>0, d=d\left(\varrho_{1}^{0}\right)>0$, and in the second matrix $f_{0^{\prime} \phi \phi}=f_{0^{\prime} \phi \phi}\left(\varrho_{2}^{0}, 1\right)>0, f_{0^{\prime} \varrho \varrho}=f_{2}^{\prime \prime}\left(\varrho_{2}^{0}\right)>0, d=d\left(\varrho_{2}^{0}\right)>0$. In case $\lambda \neq 0$ the eigenvalues and eigenvectors for the first matrix are

$$
\begin{array}{ll}
\eta_{1}=-\lambda / 2+\sqrt{\lambda^{2} / 4+f_{0^{\prime} \phi \phi}}>0, & e_{1}=\left(1, \eta_{1}, \frac{\lambda\left(\varrho_{2}-\varrho_{1}\right)}{f_{1}^{\prime \prime} d \eta_{1}}\right), \\
\eta_{2}=-\lambda / 2-\sqrt{\lambda^{2} / 4+f_{0^{\prime} \phi \phi}}<0, & e_{2}=\left(1, \eta_{2}, \frac{\lambda\left(\varrho_{2}-\varrho_{1}\right)}{f_{1}^{\prime \prime} d \eta_{2}}\right), \\
\eta_{3}=0, & e_{3}=(0,0,1),
\end{array}
$$

and for the second matrix

$$
\begin{array}{ll}
\eta_{1}=-\lambda / 2+\sqrt{\lambda^{2} / 4+f_{0^{\prime} \phi \phi}}>0, & e_{1}=\left(1, \eta_{1}, 0\right), \\
\eta_{2}=-\lambda / 2-\sqrt{\lambda^{2} / 4+f_{0^{\prime} \phi \phi}}<0, & e_{2}=\left(1, \eta_{2}, 0\right), \\
\eta_{3}=-\frac{\lambda}{f_{2}^{\prime \prime} d}, & e_{3}=(0,0,1),
\end{array}
$$

For the first matrix the signs of the eigenvalues are independent of $\lambda$. There is always one outgoing direction $+e_{1}$ at $\xi=-\infty$ with eigenvalue $\eta_{1}>0$. For the second matrix the sign of the eigenvalue 
$\eta_{3}$ depends on $\lambda$. If $\lambda>0$ there are two incoming directions $-e_{2}$ and $\pm e_{3}$ at $\xi=+\infty$ with eigenvalues $\eta_{2}<0$ and $\eta_{3}<0$. For $\lambda<0$ there is only one incoming direction $-e_{2}$ with eigenvalue $\eta_{2}<0$. This makes it plausible that in the first case one has to fit one parameter, whereas in the second two parameters are needed. This motivates the following

6.8. Conjecture. Let $f_{d}$ be as in 6.17). Then the following holds:

(1) In the region $\left\{f_{d}>0\right\}$ there exists a function $\left(\varrho_{1}, \varrho_{2}\right) \mapsto \hat{\lambda}\left(\varrho_{1}, \varrho_{2}\right)>0$ such that a trajectory of system 6.1) with (6.2) exists for all parameters $\varrho_{1}$ and $\varrho_{2}$ and with $\lambda=\hat{\lambda}\left(\varrho_{1}, \varrho_{2}\right)$.

(2) In the region $\left\{f_{d}<0\right\}$ there exists a curve $\varrho_{2}=\hat{\varrho}_{2}\left(\varrho_{1}\right)$ and on this curve a function $\varrho_{1} \mapsto$ $\hat{\lambda}\left(\varrho_{1}\right)<0$ such that a trajectory of system 6.1 with 6.2 exists for all $\varrho_{1}$ and with $\varrho_{2}=\varrho_{2}\left(\varrho_{1}\right)$ and $\lambda=\hat{\lambda}\left(\varrho_{1}\right)$.

(3) On the curve $\left\{f_{d}=0\right\}$ there exists at least one intersection point with the curve $\left\{\mu_{d}=0\right\}$. For those points the trajectory is a solution of system 6.1) with 6.2 and $\lambda=0$.

This has been confirmed by numerical simulations for the simplest case of two Gibbs free energy functions as shown in the following section.

\section{Numerical solution of the transition problem}

We investigate the nonlinear ODE system (6.1) with asymptotic boundary conditions (6.2) and (6.3) for given values of $\varrho_{1}=\varrho_{1}^{0}, \varrho_{2}=\varrho_{2}^{0}$ and $\lambda$. Assuming a monotone behaviour of $\phi$ we transform it into a nonautonomous ODE system for the phase function derivative $\psi(\phi)=\phi^{\prime}=\mathrm{d} \phi / \mathrm{d} \xi$ and the mixture concentration $\varrho=\varrho(\phi)$ as functions of the phase variable $0 \leqslant \phi \leqslant 1$ :

$$
\frac{\mathrm{d} \psi}{\mathrm{d} \phi}=\frac{B}{\psi}-\lambda, \quad \frac{\mathrm{d} \varrho}{\mathrm{d} \phi}=-\frac{C}{A}+\lambda \frac{\phi\left(\varrho_{2}-\varrho\right)}{d A \psi}
$$

with $\psi(\phi)>0$ for $0<\phi<1$ and boundary conditions

$$
\psi(0)=\psi(1)=0, \quad \varrho(0)=\varrho_{1}, \varrho(1)=\varrho_{2} .
$$

Here we assume, for simplicity, a constant diffusion coefficient $d$ and we use the following abbreviations for the coefficient functions depending on $(\phi, \varrho)$ :

$$
\begin{aligned}
& A=f_{0^{\prime} \varrho \varrho}=(1-\chi) A_{1}+\chi A_{2}>0, \\
& B=f_{0^{\prime} \phi}=(1-\chi) B_{1}+\chi B_{2}+\chi^{\prime}\left(F_{2}-F_{1}\right), \\
& C=B^{\prime} \varrho=f_{0^{\prime} \varrho \phi}=(1-\chi) C_{1}+\chi C_{2}+\chi^{\prime}\left(D_{2}-D_{1}\right),
\end{aligned}
$$

where for each phase $k$ the corresponding coefficients $D_{k}=F_{k^{\prime} \varrho}, B_{k}=F_{k^{\prime} \phi}$ and $A_{k}=D_{k^{\prime} \varrho}>0$, $C_{k}=B_{k^{\prime} \varrho}$ are directly derived from the function $F_{k}\left(\varrho, \phi_{k}\right)$ appearing in the interpolation formula (6.8) and defined by 6.9), 6.13).

The following numerical calculations are performed only for the standard example of Gibbs free energies (see Section 6.3, as plotted in Fig. 44. Then the parameter functions in 7.3 can be given explicitly:

$$
\begin{aligned}
A_{k} & \left.=f_{k}^{\prime \prime}(\varrho)=b_{k} / \varrho>0 \quad \text { (independent of } \phi\right), \\
B_{k} & =\varrho \cdot C_{k}, \\
C_{k} & \left.=b_{k} \theta_{k}^{\prime}\left(\phi_{k}\right)\left|\ln \left(1-\theta_{k}\left(\phi_{k}\right)\right)\right| \quad \text { (independent of } \varrho\right), \\
D_{k} & =b_{k}\left(\ln \frac{\varrho}{\varrho_{k}^{*}}+\beta\left(\theta_{k}\left(\phi_{k}\right)\right)\right) .
\end{aligned}
$$


However, the presented numerical procedure to solve the degenerate ODE system (7.1) and to determine characteristic properties of the phase transition layer is generally valid and essentially depends on the imposed conditions on the interpolated free energy, which are stated in 6.2 and which have to hold uniformly for compact $\varrho$-intervals in $] 0, \infty[$. Besides the convexity condition $A>0$, most important are the following conditions (fulfilled for the standard Gibbs energies 6.3):

$$
\begin{aligned}
B\left(0, \varrho_{1}\right) & =B\left(1, \varrho_{2}\right)=0, & & \\
B_{1}^{0} & =B^{\prime}{ }_{\phi}\left(0, \varrho_{1}\right)>0 & & \left(\text { Gibbs: } b_{1} s_{1}^{2} \varrho_{1}\right), \\
B_{2}^{0} & =B^{\prime}{ }_{\phi}\left(1, \varrho_{2}\right)>0 & & \left(\text { Gibbs: } b_{2} s_{2}^{2} \varrho_{2}\right) .
\end{aligned}
$$

They guarantee that for each possible solution of (7.1) with boundary conditions (7.2) the phase function derivative $\psi(\phi)$ degenerates to zero at $\phi=0$ and $\phi=1$ with positive or negative slope, respectively, corresponding to the eigenvalues $\eta_{1}>0$ in 6.21) and $\eta_{2}<0$ in 6.22, respectively,

$$
\begin{aligned}
& \eta_{1}\left(\lambda, \varrho_{1}\right)=\frac{\mathrm{d} \psi}{\mathrm{d} \phi}(0)=\sqrt{\lambda^{2} / 4+B_{1}^{0}}-\lambda / 2>0, \\
& \eta_{2}\left(\lambda, \varrho_{2}\right)=\frac{\mathrm{d} \psi}{\mathrm{d} \phi}(1)=-\left(\sqrt{\lambda^{2} / 4+B_{2}^{0}}+\lambda / 2\right)<0
\end{aligned}
$$

with both slopes $\eta_{k}$ decreasing for increasing values of the interface speed $\lambda$ that has to be determined. Moreover, at $\phi=0$ also the slope of $\varrho(\phi)$ is finite, since the $\varrho$-component of the eigenvalue $e_{1}$ in 6.21 is

$$
\eta_{1}^{\varrho}\left(\lambda, \varrho_{1}, \varrho_{2}\right)=\frac{\mathrm{d} \varrho}{\mathrm{d} \phi}(0)=\frac{\varrho_{2}-\varrho_{1}}{d A\left(0, \varrho_{1}\right)} \frac{\lambda}{\eta_{1}\left(\lambda, \varrho_{1}\right)} .
$$

It increases with $\lambda$ but increases or decreases with increasing $\varrho_{2}$ depending on whether $\lambda>0$ or $\lambda<0$. Thus, in order to construct a 3-dimensional heteroclinic orbit $\phi \mapsto(\phi, \psi(\phi), \varrho(\phi))$ connecting the two singularities $\left(0,0, \varrho_{1}^{0}\right)$ and $\left(1,0, \varrho_{2}^{0}\right)$ we can use the following

7.1. Shooting method. Let us fix $\varrho_{1}=\varrho_{1}^{0}$, the 'outer' concentration of phase 1 . Then for given parameters $\lambda$ and $\varrho_{2}$ the shooting initial direction of the trajectory at $\phi=0$ is determined by $e_{1}=$ $\left(1, \eta_{1}, \eta_{1}^{\varrho}\right)$ in 6.21), the eigenvector defining the unique tangential direction of the 1-dimensional unstable manifold at $\left(0,0, \varrho_{1}\right)$.

In the first case, $\lambda>0$, also $\varrho_{2}=\varrho_{2}^{0}$ can be fixed since the stable manifold $\mathbf{M}$ at $\left(1,0, \varrho_{2}^{0}\right)$ is 2-dimensional with tangent space spanned by the directions $-e_{2}=\left(1, \eta_{2}, 0\right)$ and the $\varrho$-axis $\pm e_{3}$, whose saddle-point type is determined by the quotient of the corresponding negative eigenvalues in 6.22 ,

$$
\kappa\left(\lambda, \varrho_{2}\right)=\frac{\eta_{3}}{\eta_{2}}=\frac{1}{d A\left(1, \varrho_{2}\right)} \frac{\lambda}{\left|\eta_{2}\left(\lambda, \varrho_{2}\right)\right|},
$$

being positive for $\lambda>0$. Then the behaviour of the $\varrho$-component, necessarily approaching $\varrho_{2}$ for $\phi_{1}=1-\phi \rightarrow 0$, can be predicted by the following asymptotic singular equation for $y\left(\phi_{1}\right)=$ $\varrho_{2}-\varrho(\phi):$

$$
y^{\prime}=\kappa \frac{y}{\phi_{1}}+\gamma \phi_{1}+\mathcal{O}\left(\phi_{1}^{2}+|y|\right)
$$

with $\gamma=C^{\prime} \phi\left(1, \varrho_{2}\right) / A\left(1, \varrho_{2}\right)$, which in the special Gibbs case is $\gamma=s_{2}^{2} \varrho_{2}>0$. Thus, for $0<\kappa<1$ the approach is with infinite slope, $\varrho(\phi)-\varrho_{2} \sim(1-\phi)^{\kappa}$, whereas for $\kappa>1$ we obtain 


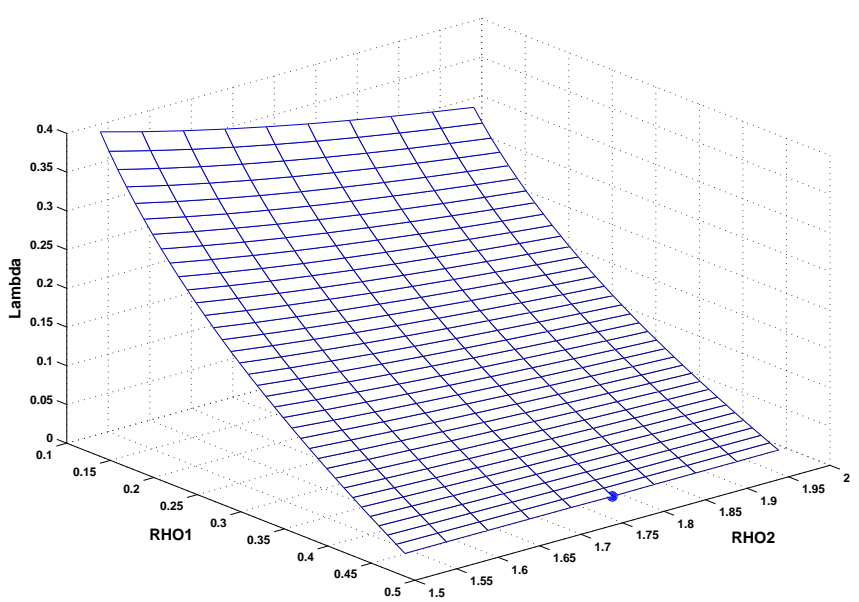

FIG. 9. Plot of $\hat{\lambda}>0$ over a $\left(\varrho_{1}, \varrho_{2}\right)$-rectangle with right hand boundary defined by $\left\{\varrho_{1} \equiv \varrho_{1}^{\text {sing }}=0.45\right\}$, thus touching the singularity (see dot) tangentially to the $f_{d}=0$ curve (see Fig. 10 below). Model parameters are the same as in Fig. 8

a zero slope with $\varrho(\phi)-\varrho_{2} \approx c \cdot(1-\phi)^{\min (\kappa, 2)}$. In cases $\kappa>2$ we can even determine the constant $c=\gamma /(\kappa-2)$, so that for $\gamma>0$ the mixture concentration $\varrho$ asymptotically approaches $\varrho_{2}$ from above. In the other cases the sign cannot be predicted beforehand.

On the other hand, for $\kappa<0$ the unique bounded solution of (7.9) shows the asymptotic behaviour $\varrho_{2}-\varrho(\phi) \sim \frac{\gamma}{2-\kappa}(1-\phi)^{2}$, so that in the case $\gamma>0$ the concentration profile approaches the value $\varrho_{2}$ from below in a quadratic monotone way. However, in general the function $\varrho(\phi)$ is not globally monotone, since according to 7.7$)$ its initial slope is proportional to $\lambda \cdot\left(\varrho_{2}-\varrho_{1}\right)$, thus with $\lambda<0$ the slope has to change sign at least once.

7.2. Iteration scheme for $\lambda>0$ (case $f_{d}>0$ ). With fixed $\varrho_{2}=\varrho_{2}^{0}$ we take $\lambda$ as shooting parameter by making use of the (numerically attested) monotonicity property, that the curve $\psi(\phi)>0$ decreases with increasing $\lambda$-values not only near $\phi=0$, but also globally. Since the first eigenvector $e_{1}$ in 6.22) with positive eigenvalue is transversal to the stable manifold $\mathbf{M}$ near the singularity $\left(1,0, \varrho_{2}\right)$, trajectories crossing the $(\psi, \varrho)$ half-plane $P^{+}=\{\phi=1, \psi>0\}$ at some positive value $\Psi_{2}(\lambda)$ are repelled by $\mathbf{M}$ with further growing $\psi$-value, thus, increasing $\lambda$ would bring the trajectory nearer to $\mathbf{M}$. On the other hand, trajectories hitting the $(\phi, \varrho)$ half-plane $P^{-}=\{\phi<1, \psi=0\}$ at some value $\Phi_{2}(\lambda)<1$ would be repelled by $\mathbf{M}$ with subsequently negative $\psi$-value, so that decreasing $\lambda$ would compensate this deviation. Therefore we can apply the following numerical iteration scheme

$$
\tilde{\lambda}=\lambda+\left\{\begin{array}{l}
R_{\psi} \Psi_{2}(\lambda)>0 \\
R_{\phi}\left(\Phi_{2}(\lambda)-1\right)<0
\end{array}\right.
$$

with suitably chosen adaptive relaxation coefficients $R_{\psi}, R_{\psi}>0$, inducing convergence to a (unique) parameter value

$$
\lambda=\hat{\lambda}\left(\varrho_{1}, \varrho_{2}\right)>0 \text {. }
$$

Let us remark that for numerical integration of the degenerate ODE system (7.1) we use an explicit forward method with adaptive step sizes $d \phi$ near $\phi=0$ and $\phi=1$, so that the initial 


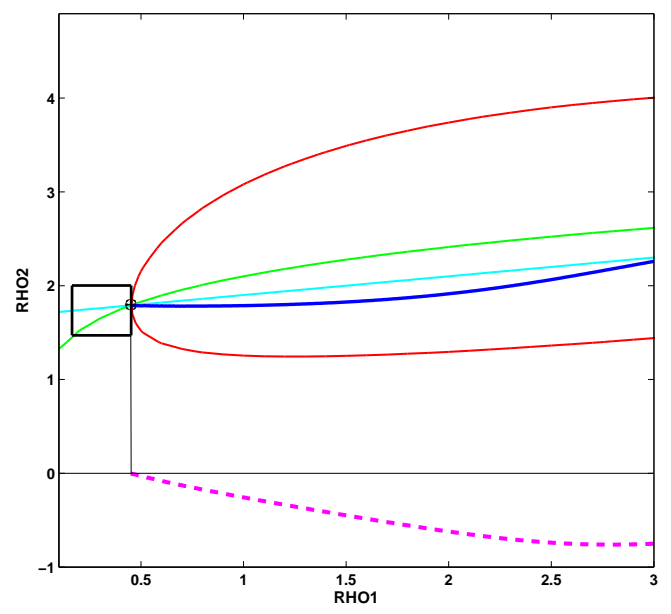

FIG. 10. Plot of the numerically computed functions $\hat{\varrho}_{2}>0$ (bold curve) and $\hat{\lambda}<0$ (dashed) as functions of $\varrho_{1}>\varrho_{1}^{\text {sing }} \approx$ 0.45 , the first approaching the singularity (dot) with $\hat{\varrho}_{2}\left(\varrho_{1}^{\text {sing }}\right)=\varrho_{2}^{\text {sing }} \approx 1.79$. The other (light) plots are the zero-level curves as in Fig. 8 Notice that the $\hat{\varrho}_{2}$ curve lies completely in the region $\mu_{d}<0$ and, at least for $\varrho_{1}<3$, also in the region $p_{d}<0$. Moreover, the rectangle in the $f_{d}>0$ region touching the singularity defines the domain over which $\hat{\lambda}\left(\varrho_{1}, \varrho_{2}\right)>0$ is plotted in Fig.9

values are given by $\psi(d \phi)=\eta_{1} \cdot d \phi$ and $\varrho(d \phi)=\varrho_{1}+\eta_{1}^{\varrho} \cdot d \phi$. Simultaneously we solve the ODE for the inverse function $\xi=\xi(\phi)$, namely $\mathrm{d} \xi / \mathrm{d} \phi=1 / \psi(\phi)$ with fixed initial condition $\xi(0)=\xi_{0} \ll 0$ as an approximation of the asymptotic condition $\phi(-\infty)=0$. Thus, within the same numerical procedure also the retransformed $\xi$-profiles of $\phi$ and $\varrho$ are obtained, being defined for $\xi_{0} \leqslant \xi \leqslant \xi_{1}=\xi(1)$ with $\phi\left(\xi_{1}\right)=1$, reflecting the asymptotic condition $\phi(\infty)=1$.

This finite numerical scheme does not always fulfill the other asymptotic condition $\varrho(\phi=1)=$ $\varrho_{2}$, but only up to a (usually small) deviation $\delta_{\varrho}$. However, due to the equation 7.9 the appearing gap can be consistently closed by approximately defining $\varrho(\xi)=\varrho_{2}+\delta_{\varrho} \exp \left(-\kappa_{\varrho}\left(\xi-\xi_{1}\right)\right)$ for $\xi \geqslant \xi_{1}$ with $\kappa_{\varrho}=\kappa /\left(d A\left(1, \varrho_{2}\right)\right.$ ) (see the plot of $\varrho$ in Fig. 13k with $\left.\xi_{1}=1.1\right)$. Notice that this approximation is valid only for cases where $\left|\delta_{\varrho}\right|$ is relatively small and the positive 'saddle type' index $\kappa \sim \lambda$ in 7.8 is not too small. Other situations cannot be handled so far, which particularly occur if $\lambda=\hat{\lambda}\left(\varrho_{1}, \varrho_{2}\right)>0$ converges to zero as the point $\left(\varrho_{1}, \varrho_{2}\right)$ approaches the $\left\{f_{d}=0\right\}$ curve in Fig. 8 from the left, but outside the singularity.

7.3. Iteration scheme for $\lambda<0$ (case $f_{d}<0$ ). In this case we have to take $\lambda<0$ and $\varrho_{2}>0$ as shooting parameters, since due to $\kappa<0$ in 7.8 we now have two positive eigenvalues in 6.22) and an only 1-dimensional stable manifold $\mathbf{M}$ near the singularity $\left(1,0, \varrho_{2}\right)$ with unique tangent direction $-e_{1}=\left(-1,\left|\eta_{2}\right|, 0\right)$. Because the other, repelling eigenvectors $e_{2}$ and $e_{3}$ are orthogonal, the following extended iteration scheme appears to be appropriate: As in the previous case, we determine either (if hitting $P^{+}$) the trajectory components at $\phi=1$, namely $\Psi_{2}\left(\lambda, \varrho_{2}\right)=\psi(1)>0$ and $\mathcal{P}_{2}\left(\lambda, \varrho_{2}\right)=\varrho(1)$ or (if hitting $P^{-}$) the value $\phi=\Phi_{2}\left(\lambda, \varrho_{2}\right)<1$, at which $\psi(\phi)=0$ as well as $\mathcal{P}_{2}\left(\lambda, \varrho_{2}\right)=\varrho(\phi)$. Then we set the iterated value $\tilde{\lambda}$ as before in $(7.10)$ and, in addition,

$$
\tilde{\varrho}_{2}=\varrho_{2}+R_{\varrho}\left(\mathcal{P}_{2}\left(\lambda, \varrho_{2}\right)-\varrho_{2}\right),
$$

again by choosing suitable adaptive relaxation coefficients resulting in convergence of this iteration 
scheme to (unique) values

$$
\lambda=\hat{\lambda}\left(\varrho_{1}\right)<0, \quad \varrho_{2}=\hat{\varrho}_{2}\left(\varrho_{1}\right)>0 .
$$

For the $\varrho_{2}$-iteration we again make use of the (numerically attested) global monotonicity property that is carried over from the local property stated after (7.7). Varying the $\varrho_{1}$ value above $\varrho_{1}^{\text {sing }}$ and determining the solutions of (7.13) yields a curve $\varrho_{2}=\hat{\varrho}_{2}\left(\varrho_{1}\right)$ that lies in the $\left\{f_{d}<0\right\}$ region and converges to the singular value $\hat{\varrho}_{2}=\varrho_{2}^{\text {sing }}$ for $\varrho_{1} \rightarrow \varrho_{1}^{\text {sing }}$ (see Fig. 10). Moreover, along this determined 'jump curve' for the sharp interface of a relatively shrinking disordered phase, the condition $\mu_{d}<0$ always holds. According to definition (6.18) this means that the interface always experiences a downward jump of the potential $\mu_{1}\left(\varrho_{1}\right)$ to a lower potential $\mu_{2}\left(\varrho_{2}\right)$ driving the phase transition to the growing ordered phase. For $\varrho_{1}<3$ there is even a downward jump of the 'disordered pressure' $p_{1}\left(\varrho_{1}\right)$ towards a lower 'ordered pressure' $p_{2}\left(\varrho_{2}\right)$.

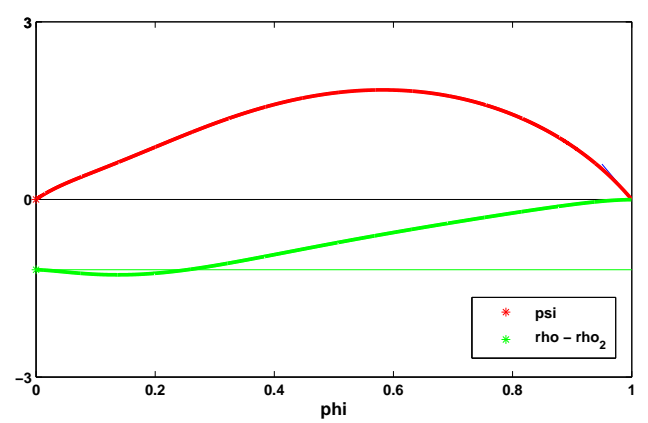

FIG. 11. Solution of the transition problem (7.1)-7.2 obtained by the shooting method for the case $f_{d}<0$ with given disordered phase level $\varrho_{1}=0.6$ just above the singularity value. Computed parameters are speed $\lambda=-0.0801$ and ordered phase level $\varrho_{2}=1.7843$. Lower curve: plot of $\varrho(\phi)-\varrho_{2}$ with $\varrho(\phi)$ connecting $\varrho_{1}$ to $\varrho_{2}$. Upper curve: $\psi(\phi)>0$ with zero values at the end points but with nonzero slopes.

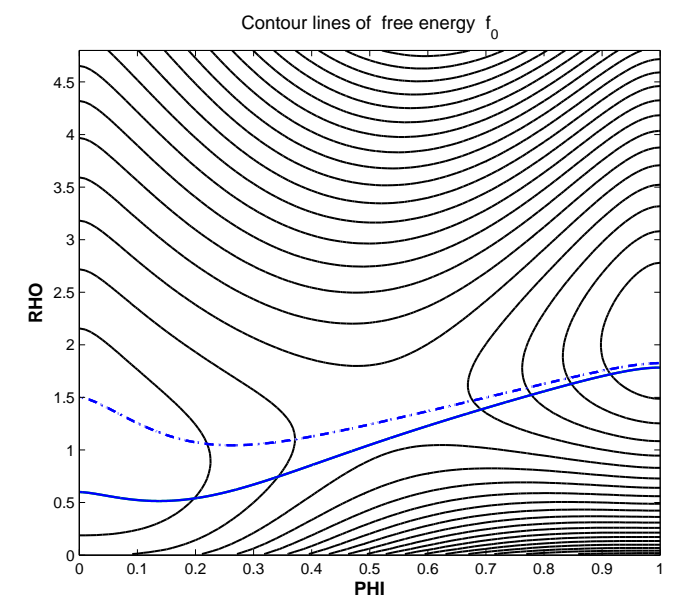

FIG. 12. Plots of two density functions $\varrho(\phi)$ of Fig. 11 into the contour map of the free energy $f_{0}$ in Fig. 6 for the case $f_{d}<0$ with $\varrho_{1}=0.6$ (full curve) and $\varrho_{1}=1.5$ (dashed curve). 
Fig. 11 shows an example of such a solution trajectory obtained by the shooting method. The $\varrho(\phi)$ curve appearing there is also plotted into the contour map of the free energy function (see Fig. 12, together with a second one for a larger $\varrho_{1}$-value. Both curves represent saddle-point transition paths that quite well experience lowest free energy levels.

(a)

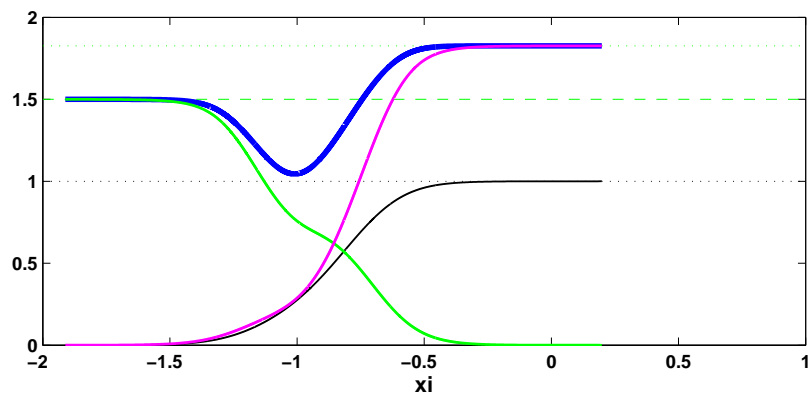

(b)

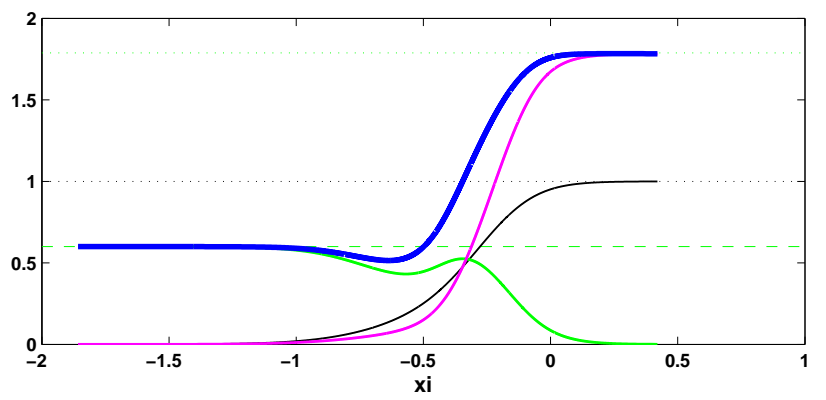

(c)

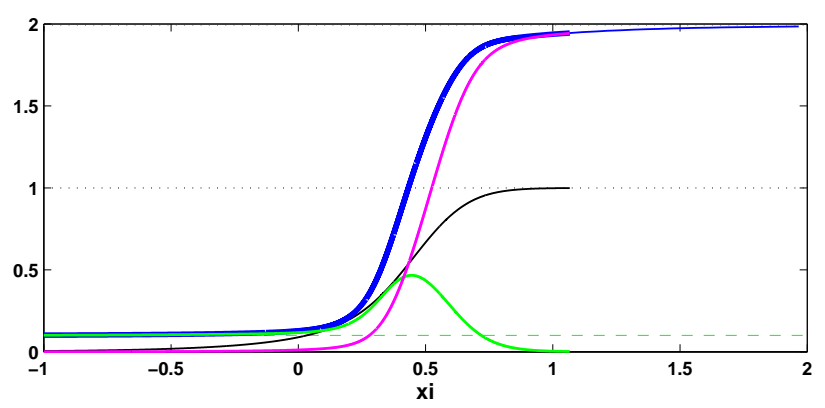

FIG. 13. Plotted over the spatial transition coordinate $\xi$ are the phase function $\phi(\xi)$ (thin curve) and the density functions $\varrho(\xi)$ (very fat curve), as well as the specific phase densities $\varrho_{1}(\xi)=(1-\phi(\xi)) \cdot \varrho(\xi)$ and $\varrho_{2}(\xi)=\phi(\xi) \cdot \varrho(\xi)$ for the following parameter choices: (a) $\varrho_{1}=1.5$ with computed $\lambda=-0.4485$, (b) $\varrho_{1}=0.6$ corresponding to Fig. 11 with $\lambda=-0.080$, and (c) $\varrho_{1}=0.1$ and $\varrho_{2}=1.9$ with positive $\lambda=0.3043$.

In all situations of a growing ordered phase $(\lambda<0)$ the $\varrho_{2}(\xi)$-profile is monotone increasing with steeper slope, the lower the absolute value $|\lambda|$ of the local interface speed is (see Fig. 13). For larger $\varrho_{1}$, also the $\varrho_{1}(\xi)$-profile is monotone (see Fig. 13 ). Indeed, it strongly decreases already at low $\phi(\xi)$-amounts of ordered lipids, where interactions are negligible, so that the disordered lipids can diffuse (though with a relatively low value, here $d=0.05$ ) into the region $\phi \approx 1 / 2$, 
where transition into ordered lipids is prevalent. This effect induces a nonmonotone mixture concentration profile $\varrho(\xi)$ with a depletion minimum at $\phi \approx 1 / 4$. On the contrary, for smaller $\varrho_{1}$ the $\varrho_{1}(\xi)$-profile reveals a more complex transition dynamics (Fig. 13p). The initial decrease due to diffusion is obvious. However, for low growth speed $(|\lambda| \approx 0.08)$ and correspondingly low 'feeding' concentration $\left(\varrho_{1}^{0}=0.6\right)$, the $\varrho_{1}(\xi)$-profile expresses a distinguished hump near the region $\phi(\xi) \approx 1 / 2$ with a peak value as high as $\varrho_{1}^{0}$. The same behaviour, even more expressed, is seen in situations of a shrinking ordered phase $(\lambda>0)$ (see Fig. 13 k). Therefore we conclude that the hump is due to ordered lipids that underwent fast transition to a disordered state in a region where diffusion is still negligible according to definition (5.3.

\section{Numerical solution of the interface problem}

In the simple 1-dimensional case of $\Omega=\left[0, \infty[\times] 0, L\left[\right.\right.$, that is, a fixed interval $\left.\Omega_{t}=\right] 0, L[$ for $t>0$, we study the dynamical behaviour of the sharp interface problem $(3.7)-(3.12)$ with just one transition jump at the moving interface $\Gamma=\{(t, x): x=s(t)\}$ between the disordered phase density $\varrho_{1}$ on $\left.\Omega_{t}^{1}=\right] 0, s(t)\left[\right.$ and the ordered phase density $\varrho_{2}$ on $\left.\Omega_{t}^{2}=\right] s(t), L[$. According to the definition of $\lambda$ in 3.9 the net speed of the free boundary $\Gamma_{t}$ is

$$
\dot{s}:=\lambda+V, \quad V(t)=v(t, s(t)),
$$

where $V$ denotes the velocity of the bulk flow at the interface, relative to which the function $\lambda$ represents the interface speed. We assume that the coefficients of $\Pi^{k}$ are dominating, that is, the lipid viscosity and pressure terms dominate all forces due to inertia or diffusion. Then equation (3.4) becomes

$$
\operatorname{div} \sum_{k=1,2} \Pi^{k} \mathbf{m}_{\Omega^{k}}=0
$$

that is, the pressure tensor in $1 \mathrm{D}$ is constant, $\Pi^{1}=\Pi^{2}=$ const. With a constant viscosity parameter $\alpha_{k}>0$ we therefore conclude

$$
p_{k}\left(\varrho_{k}\right)-\alpha_{k} \cdot \partial_{x} v=\Pi^{k}=\mathrm{const} \quad \text { for } k=1,2 .
$$

No-flux conditions at the fixed boundaries then give an explicit expression for the viscous flow at the interface:

$$
V(t)=\frac{\frac{1}{s(t)} \int_{0}^{s(t)} p_{1}\left(\varrho_{1}(t, x)\right) \mathrm{d} x-\frac{1}{L-s(t)} \int_{s(t)}^{L} p_{2}\left(\varrho_{2}(t, x)\right) \mathrm{d} x}{\frac{\alpha_{1}}{s(t)}+\frac{\alpha_{2}}{L-s(t)}},
$$

which is proportional to the difference between the mean pressures in the two phases. Thus, it remains to numerically solve the pair of parabolic and hyperbolic equations 3.7) for $\varrho_{1}(t, x)$ and $\varrho_{2}(t, x)$ with diffusive flux $J=-d \cdot \partial_{x} f_{1}^{\prime}\left(\varrho_{1}\right)=-\frac{d b_{1}}{\varrho_{1}} \partial_{x} \varrho_{1}$ in (3.3) coupled with the jump condition (3.8) at the interface, yielding a Neumann boundary condition for $\varrho_{1}$ :

$$
\frac{d b_{1}}{\varrho_{1}} \partial_{x} \varrho_{1}+\lambda \cdot\left(\varrho_{1}-\varrho_{2}\right)=0 \quad \text { on } \Gamma=\{(t, x): x=s(t)\} .
$$


In cases of $f_{d}\left(\varrho_{1}^{0}, \varrho_{2}^{0}\right)<0$, with $\varrho_{1}^{0}(t)=\varrho_{1}(t, s(t))$ and $\varrho_{2}^{0}(t)=\varrho_{2}(t, s(t))$, it follows that $\lambda=\hat{\lambda}\left(\varrho_{1}^{0}\right)<0$, therefore by $(8.1)$ the bulk velocity satisfies $V>\dot{s}$, so that at the phase boundary the characteristics of the hyperbolic equation point into the ordered phase domain $\Omega^{2}$, requiring the prescription $\varrho_{2}^{0}=\hat{\varrho}_{2}\left(\varrho_{1}^{0}\right)$ given in (7.13). Otherwise, if $f_{d}\left(\varrho_{1}^{0}, \varrho_{2}^{0}\right)>0$ then $\lambda=\hat{\lambda}\left(\varrho_{1}^{0}, \varrho_{2}^{0}\right)>0$. Hence $\lambda$ depends on the resulting two boundary densities of the coupled parabolic and hyperbolic equations.

In order to avoid initial transients, already the initial values $\varrho_{1}(0, x)$ and $\varrho_{2}(0, x)$ should satisfy the jump condition (8.4), and in the case $\lambda<0$ also (7.13). Then the slope $\partial_{x} \varrho_{1}(0, s(0))$ at the interface is determined. By prescribing the initial size $s(0)=s_{0}<L$ of the disordered phase and choosing a suitable initial $\varrho_{1}$-profile, we fix the total mass

$$
M_{\varrho}=\int_{0}^{s(t)} \varrho_{1}(t, x) \mathrm{d} x+\int_{s(t)}^{L} \varrho_{2}(t, x) \mathrm{d} x
$$

of lipids in the interval $[0, L]$, which is preserved over time. Notice that in the case of asymptotic convergence to the steady state solution $\left(\varrho_{1} \equiv \varrho_{1}^{\text {sing }}, \varrho_{2} \equiv \varrho_{2}^{\text {sing }}\right)$ the final position of the interface $s(\infty)$ is given by

$$
s(\infty)=\frac{L \cdot \varrho_{2}^{\text {sing }}-M_{\varrho}}{\varrho_{2}^{\text {sing }}-\varrho_{1}^{\text {sing }}},
$$

yielding the restriction $\varrho_{1}^{\text {sing }} \cdot L<M_{\varrho}<\varrho_{2}^{\text {sing }} \cdot L$, which must hold for such a convergence to happen.

Indeed, $\left(\varrho_{1}^{\text {sing }}, \varrho_{2}^{\text {sing }}\right)$ is the unique one-jump plateau solution satisfying all steady state conditions, since $v \equiv 0$ and $\dot{s}=0$ with 8.1 -8.4 imply $V=0$ and $\lambda=0$, thus also $p_{d}=0$ and $f_{d}=0$. This is in accordance with the assertion in 6.8 3 that for $\lambda=0$ transitions are only possible at intersection points of these two zero level curves (see Fig. 10p. Depending on the two free energy functions, there may exist a second intersection point which, however, has very large jump densities and probably is unstable under the outer dynamics.

On the contrary, local linearized analysis around $\left(\varrho_{1}^{\text {sing }}, \varrho_{2}^{\text {sing }}\right)$ of the simple 'outer' dynamics defined above leads to an ODE system for $\varrho_{1}^{0}(t), \varrho_{2}^{0}(t)$ and the interface position $s(t)$ revealing asymptotic stability for every final jump position $s(\infty)$, however, with different exponential convergence rates for solutions with $\lambda>0$ and $\lambda<0$. These properties are reproduced by the global behaviour of the following numerical simulations.

8.1. Dissolving LC domain of ordered lipids (case $\lambda>0$ ). Fig. 14 shows the 'jump density trajectories' of two solutions with the same initial values $s_{0}=0.5$ and $\varrho_{1}^{0}=0.3$ but with different $\varrho_{2}^{0}$-levels 1.65 and 1.9. The trajectories asymptotically approach each other quite fast (after $t \sim$ $1 \mathrm{sec})$ and then slowly converge towards the singularity $\left(\varrho_{1}^{\text {sing }}, \varrho_{2}^{\text {sing }}\right)$. For $\varrho_{2}^{0}=1.65$ the size of the disordered LE domain, $s(t)$, slightly increases with time and stabilizes at $s(\infty) \approx 0.55$. However, for $\varrho_{2}^{0}=1.9$, the initial LC domain $\left.\Omega_{0}^{2}=\right] s_{0}, L$ [ carries a high internal pressure, because $p_{d}>0$ means $p_{2}\left(\varrho_{2}^{0}\right)>p_{1}\left(\varrho_{1}^{0}\right)$. Therefore, initially the bulk velocity $V<0$ at the interface exceeds the relative transition speed $\lambda>0$ leading to a negative interface speed $\dot{s}=V+\lambda<0$, i.e. expansion of the LC domain for $t<0.3 \mathrm{sec}$ (see Fig. 15) before it later starts to shrink. 


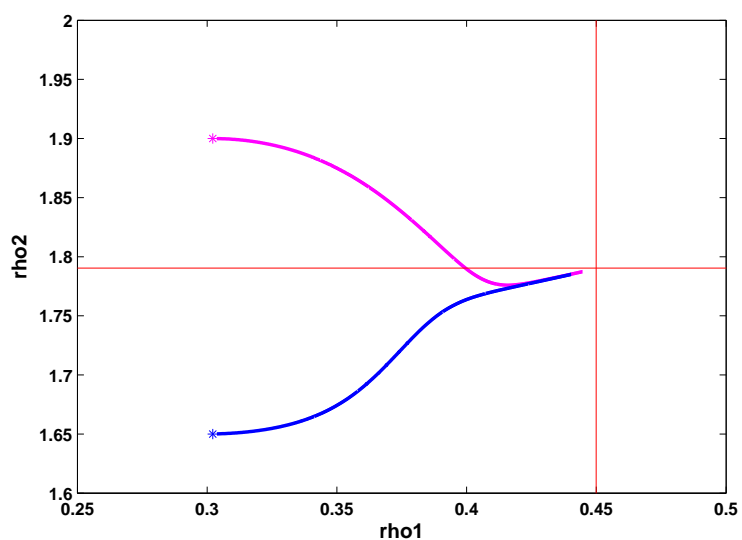

FIG. 14. Solution of the 1-dimensional interface dynamics over a fixed interval of length $L=1$ in the case $\lambda>0$ : The time trajectory of the density jump pair $\left(\varrho_{1}^{0}(t), \varrho_{2}^{0}(t)\right)$ with $0 \leqslant t \leqslant 3$ is plotted for two different initial values: $\varrho_{1}^{0}(0)=0.3$, $\varrho_{2}^{0}(0)=1.65$ (lower curve) and 1.9 (upper curve), moreover $s_{0}=s(0)=0.5$. For $t>1$ the two trajectories coincide and slowly converge to the unique steady state, whose density jump is given by $\left(\varrho_{1}^{\operatorname{sing}}, \varrho_{2}^{\operatorname{sing}}\right)$. Comparison with Fig. 8 shows that the lower trajectory always satisfies the pressure jump condition $p_{d}<0$, whereas the upper trajectory first starts in the region $p_{d}>0$. Model parameters are as before, with additional viscosities $\alpha_{1}=0.4$ in the disordered phase and $\alpha_{2}=1$ in the ordered phase.

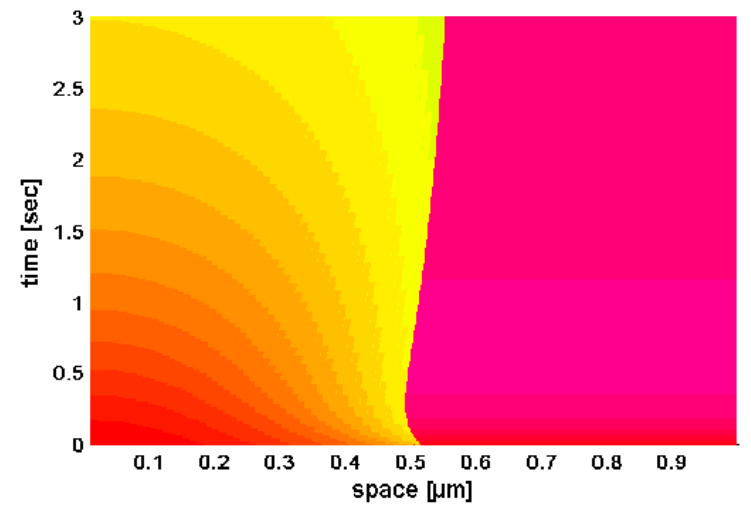

FIG. 15. Space-time color-coded density plot of the two-phase dynamics for the upper trajectory in Fig. 14 showing the sharp interface $x=s(t)$ between the disordered LE domain (left) and the ordered LC domain (right), which first shrinks and then expands.

Indeed, the whole initial velocity profile in Fig. 16 is negative, and it stays linear in the LC domain, meaning that the initial LC phase expansion is mainly due to a viscous divergence of the plateau $\varrho_{2} \equiv \varrho_{2}^{0}(t)$, thereby decreasing in time. Later (see Fig. 16 p for $t=1$ ) the situation is reversed: The ongoing transition of ordered lipids at the interface into the disordered phase $(\lambda>0)$ is dominant so that the $\mathrm{LC}$ domain shrinks, but also due to a slight viscous compression indicated by the positive value $V>0$. The observed negative bulk velocity $v$ in the growing LE domain $\Omega_{t}^{1}=$ ]0, $s(t)$ [ is due to viscous flux going in the same (negative) direction as the diffusive flux, namely away from the interface, where new disordered lipids are 'generated' according to the microscopic transition layer profile visualized in Fig. 13 . 
(a)
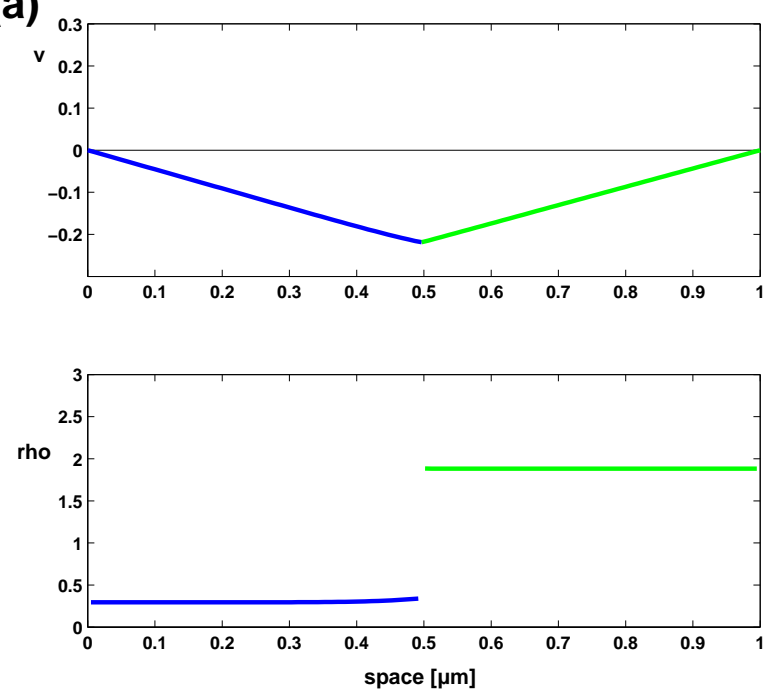

(b)
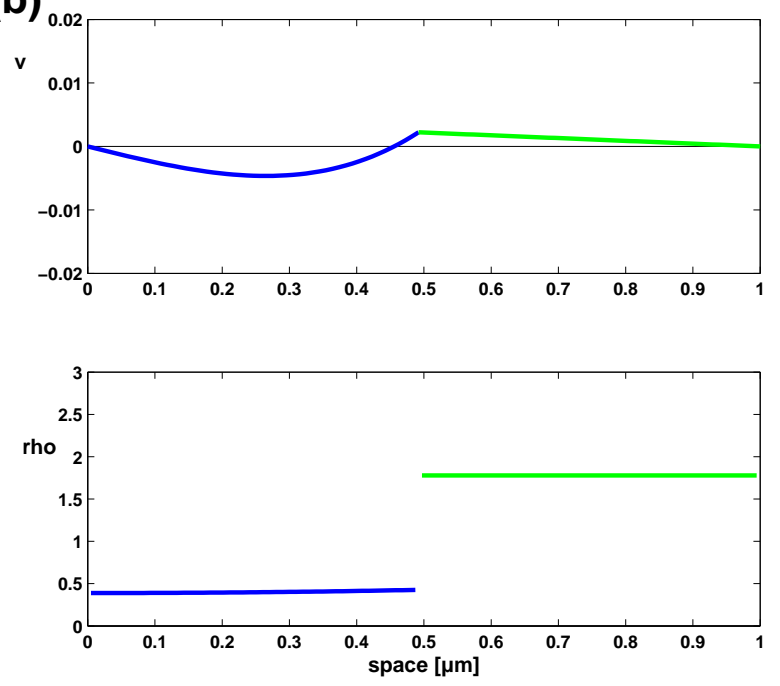

FIG. 16. Plot over space coordinate $x$ of the bulk velocity $v(t, x)$ (upper panel) as computed by 8.2 and of the density profile $\varrho(t, x)$ (lower panel) for the upper trajectory in Fig.14 (a) Initial profiles at $t=0$, and (b) profiles at $t=1$.

8.2. Expanding LC domain (case $\lambda<0$ ). Fig. 17 shows the spatiotemporal plot of the sharp interface dynamics in the case $\lambda<0$, where the ordered lipid domain is slowly expanding due to transition and uptake of disordered lipids at the interface. Nevertheless, the initial negative jump with a higher density $\varrho_{1}^{0}>\varrho_{2}^{0}$ at the interface induces a strong diffusive flux away from the interface which, however, soon reverses to the canonical situation of a positive jump (for times $t>0.05 \mathrm{sec}$ ). The expanding LC domain does not promptly reach the slightly decreasing plateau level in regions 


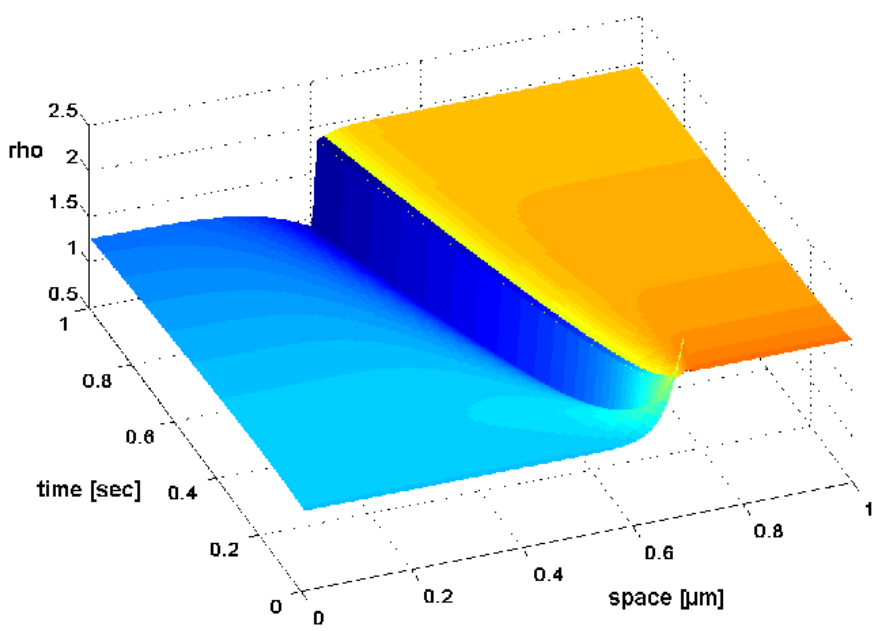

FIG. 17. Spatiotemporal density plot for the 1D sharp interface dynamics in the case $\lambda<0$, with initial jump densities $\varrho_{1}^{0}(0)=2.7$ given and $\varrho_{2}^{0}(0)=\varrho_{2}\left(\varrho_{1}^{0}(0)\right)=2.14<\varrho_{1}^{0}(0)$, moreover $s_{0}=0.7$. Other parameters are as in the figures before.

behind the interface because of viscosity. For very long times we again observe a slow convergence to the singular plateau solution $\left(\varrho_{1}^{\text {sing }}, \varrho_{2}^{\text {sing }}\right)$ with $s(\infty) \approx 0.3$.

For the same initial density jump at the interface, but now with relatively high $\varrho_{1}$ concentration in the LE domain, such that the total mass satisfies $M_{\varrho}>L \cdot \varrho_{2}^{\text {sing }}$ giving a negative value of $s(\infty)$ in 8.5 , the expansion speed of the LC domain is much increased due to more disordered lipids available for transition at the interface (see Fig. 18p). No convergence to the singularity plateau jump solution is possible in this case, rather the interface position $s(t)$ will reach zero in finite time, so that the disordered LE domain disappears and, finally, a constant ordered LC domain with plateau value $\varrho_{2}^{\infty}=M_{\varrho} / L$ will form. During this fast expansion mode the corresponding profiles in Fig. 18 (for $t=1 \mathrm{sec}$ ) again reveal not only a diffusive flux of disordered lipids towards the growing interface, but also an associated positive viscous flux, even up to the interface, behind which the ordered lipids of the newly formed LC domain stay under viscous compression in a certain boundary region. The remaining part of the LC expanding domain, however, shows a divergent (negative) viscous flux due to a slight lowering of the $\varrho_{2}$ plateau level with time.

The results obtained here from exemplary numerical simulation of the sharp interface dynamics in Section 3 by using the transition layer analysis for interpolated Gibbs free energies and a most simplified 1-dimensional global boundary value problem for one-jump solutions, confirm the important role of the singularity $\left(\varrho_{1}^{\text {sing }}, \varrho_{2}^{\text {sing }}\right)$ of jump densities at the interface, which had been indicated already by analytical means. In both cases $\lambda>0$ (Fig. 14) and $\lambda<0$ (Figs. 10 and 17p under the condition $0<s^{\infty}<L$ the jump pair trajectories asymptotically converge to the singularity, but with different convergence rates and jump relations. Indeed, whether $\varrho_{1}^{0}(t)$ approaches $\varrho_{1}^{\text {sing }}$ from below or above, for large times $\varrho_{2}^{0}(t)$ is always increasing towards $\varrho_{2}^{\text {sing }}$, though very slowly in the latter case. 

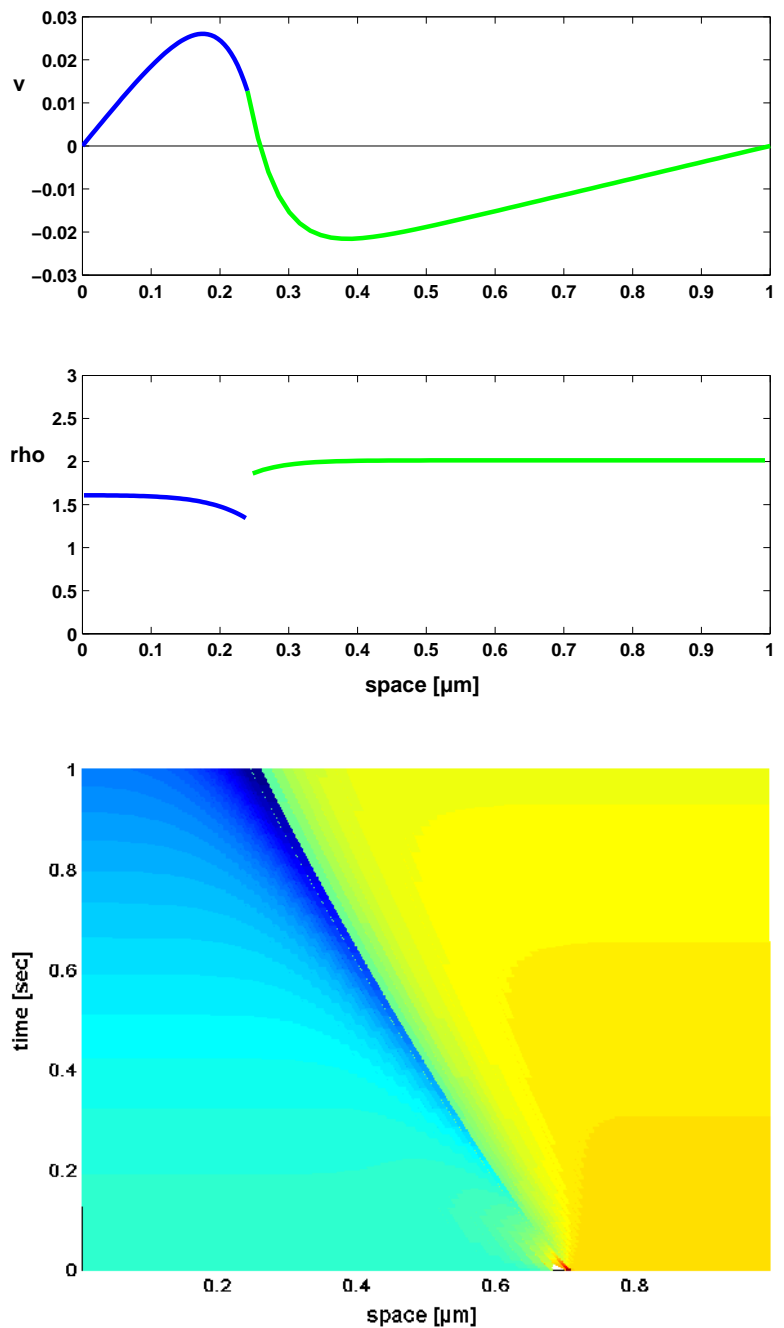

FIG. 18. Interface dynamics for the same initial conditions as in Fig. 17 but with increased mass of disordered lipids in the initial LE domain $\left.\Omega_{0}^{1}=\right] 0, s_{0}[$. (a) Velocity and density profiles as in Fig. 16 at the end of simulation time $(t=1)$; (b) corresponding space-time plot as in Fig. 15 now revealing a fast expansion of the LC domain $\left.\Omega_{t}^{2}=\right] s(t), L[$.

\section{Conclusion}

We model the surfactant layers on top of the thin water film covering the interior of lung alveoli. We consider two main phases of interacting bio-molecules, a disordered diffusive and an ordered highly viscous phase (see Sections 2 and 3). This type of model has not been treated before (see [2] for a review of diffusive interface models).

We develop a new phase transition model (see Sections 4 and 5 ) in order to derive a second free boundary condition for the sharp interface model. This phase transition model depends on a small parameter $\varepsilon$, which approximately is the thickness of the transition layer between disordered phase 
$l$ with density $\varrho_{1}$ and ordered phase 2 with density $\varrho_{2}$. Here the $\phi$-equation results from a scaling that is different from the usual Allen-Cahn procedure. In the ordered phase a small diffusion term is introduced, which is proportional to the same parameter $\varepsilon$. Thus the transport equations on the whole domain are a parabolic regularization.

Our method essentially depends on thermodynamical arguments, which require an inequality for the free energy production. This leads to a phase transition model for the partition functions $\phi_{i}=$ $\varrho_{i} / \varrho$ with mixture density $\varrho=\varrho_{1}+\varrho_{2}$. This model depends on the following assumptions for the free energies and the diffusion fluxes: (a) appropriate interpolation hypotheses on the homogeneous part of the free energy, which are explicitly modelled using microscopic considerations and chemical transition kinetics, e.g. of Monod type; (b) simple decomposition of the diffusive flux

$$
J=-d_{\varepsilon}(\varrho, \phi) \nabla\left(f_{0^{\prime} \varrho}\right)=J_{1}+J_{2} \quad \text { with } J_{k}=\phi_{k} J ;
$$

(c) a generally nonlinear interpolation of the inverse diffusion coefficients

$$
\frac{1}{d_{\varepsilon}(\varrho, \phi)}=\frac{1}{d(\varrho)} h\left(\phi_{1}\right)+\frac{1}{\varepsilon d(\varrho)} h\left(\phi_{2}\right)
$$

For the formal asymptotic limit, as $\varepsilon \rightarrow 0$, we obtain a transition layer, which is a second order ODE for the partition function $\phi=\phi_{1}$ plus a first order equation for $\varrho$ with asymptotic values $\varrho_{1}^{0}$ and $\varrho_{2}^{0}$ characterizing the jumping densities of the macroscopic sharp interface problem.

In order to determine the free parameter $\lambda=\left(v_{\Gamma}-v\right) \bullet v$, the relative interface speed, we had to find the heteroclinic transition orbits of this ODE system. An indicator function $f_{d}\left(\varrho_{1}^{0}, \varrho_{2}^{0}\right)$ explicitly defined in terms of the two free energies $f_{1}$ and $f_{2}$ determines the sign of $\lambda$, thereby deciding the type of the transition dynamics and the boundary condition for the sharp interface problem: In case $f_{d}\left(\varrho_{1}^{0}, \varrho_{2}^{0}\right)>0$ both boundary values $\varrho_{k}^{0}$ are obtained by solving the system of a hyperbolic equation in the ordered phase and a parabolic equation in the disordered phase, with Neumann boundary condition containing the function $\lambda=\hat{\lambda}\left(\varrho_{1}^{0}, \varrho_{2}^{0}\right)>0$, which has to be computed. On the contrary, in case $f_{d}\left(\varrho_{1}^{0}, \varrho_{2}^{0}\right)<0$ the ordered phase density value $\varrho_{2}^{0}=\hat{\varrho}_{2}\left(\varrho_{1}^{0}\right)$ together with $\lambda=\hat{\lambda}\left(\varrho_{1}^{0}\right)<0$ has to be computed for given $\varrho_{1}^{0}$, so that $\varrho_{2}^{0}$ serves as Dirichlet boundary value for the hyperbolic problem, whose characteristics at the interface point into the ordered phase domain.

Furthermore, numerical simulations of the 1-dimensional sharp interface problem have been performed in the simplest case of a single jump between the densities in a disordered LE domain and an ordered LC domain (e.g. applied to amphiphilic lipids on a water-air surface). For a fixed interval of length $L$ with no flux boundary conditions and with different constant viscosities in the two phases, the simulations reveal that appropriately defined initial density profiles asymptotically converge towards a distinguished one-jump plateau $\left(\varrho_{1}^{\text {sing }}, \varrho_{2}^{\text {sing }}\right)$, which as steady state solution is uniquely determined by the given total lipid mass $M_{\varrho}$, provided that the latter satisfies the condition $\varrho_{1}^{\text {sing }}<M_{\varrho} / L<\varrho_{2}^{\text {sing }}$.

The stability of this steady state can be confirmed locally, but obviously holds also globally in this simple situation (as numerical simulations show). This is in favour of a biologically evolved and controlled function of surfactant patches, which also have been observed in laboratory experiments with different sign between the two plateau densities under different external pressures (see Fig.11A and 1 B). The biological function of the disordered LE domains would be to serve as buffers between the more effective surfactant monolayers of the ordered LC patches, particularly under conditions of fast compression during breathing cycles (see [3] and [9]). 
Moreover, the numerical analysis should be carried over to more appropriate model functions for the free energy of lipid monolayers, particularly for the ordered LC phase as indicated by the proposed function in Fig. 4p. Finally, an outstanding problem is to generalize the stability results to 2-dimensional geometries (see [6] for a study using nonlocal electrostatic interaction energies). Boundary regularity of growing or shrinking LC patches has to be investigated under realistic conditions, which simulate the dynamics of surfactant layers on the alveolar lung surfaces or equivalent test bubbles during experimentally applied cycles of surface compression and relaxation mimicking the breathing cycle.

\section{REFERENCES}

1. Alt, H. W. The entropy principle for interfaces. Solids and fluids. Preprint SFB 611 Univ. Bonn (2009).

2. Anderson, D. M., McFadden, G. B., \& Wheeler, A. A. Diffuse-interface methods in fluid mechanics. Ann. Rev. Fluid Mech. 30 (1998), 139-165.

3. Crane, J. M., \& HALl, S. B. Rapid compression transforms interfacial monolayers of pulmonary surfactant. Biophys. J. 80 (2001), 1863-1872.

4. Ding, J., Takamoto, D. Y., von Nahmen, A., Lipp, M. M., Lee, K. Y. C., Waring, A. J., \& ZASADZINSKI, J. A. Effects of lung surfactant proteins, SP-B and SP-C, and palmitic acid on monolayer stability. Biophys. J. 80 (2001), 2262-2272.

5. Knecht, V., Müller, N., Bonn, M., Marrink, S.-J., \& Mark, A. E. Simulation studies of pore and domain formation in a phospholipid monolayer. J. Chem. Phys. 122 (2005), 024704.

6. McConnell, H. M. Structures and transitions in lipid monolayers at the air-water interface. Ann. Rev. Phys. Chem. 42 (1991), 171-195.

7. MÜLleR, I. Thermodynamics. Pitman (1985). Zbl 0637.73002

8. SchÜrch, S., SchÜrch, D., Curstedt, T., \& Robertson, B. Surface activity of lipid extract surfactant in relation to film area compression and collapse. J. Appl. Physiol. 77 (1994), 974-986.

9. Yau, W., Piknova, B., \& Hall, S. B. The collapse of monolayers containing pulmonary surfactant phospholipids is kinetically determined. Biophys. J. 89 (2005), 306-314. 